\title{
Apparent disagreement between cyclic voltammetry and electrochemical impedance spectroscopy explained by time-domain simulation of constant phase elements
}

\author{
William J F Gannon ${ }^{\mathrm{a}}$, Charles W Dunnill ${ }^{\mathrm{a}, *}$ \\ ${ }^{a}$ Energy Safety Research Institute, Swansea University, SA1 8EN, UK
}

\begin{abstract}
A selection of electrodes was analysed using cyclic-voltammetry (CV) and electrochemical impedance spectroscopy (EIS), and a large apparent resistance was observed with $\mathrm{CV}$ that was absent with EIS. The explanation for this resistance anomaly was traced to the constant phase element (CPE) behaviour which is exhibited by the electrode double-layer capacitance. Computer simulations of the transient-response of an RQ network (where Q represents a CPE) to a voltage ramp revealed bi-exponential behaviour, with two separate time-constants. One is equal to the product of $\mathrm{R}$ and $\mathrm{Q}$, but the other is fixed at about 0.3 seconds. This finding is supported by observation, by mathematical derivation, and by a novel mixed-domain five-component equivalent circuit model. In addition, example code is provided as a basis for transient simulations of constant phase elements with arbitrary voltage waveforms. This explanation assists in the correct interpretation of potentially misleading cyclic voltammetry results.
\end{abstract}

Keywords: cyclic voltammetry, electrochemical impedance spectroscopy, constant phase element, equivalent circuit model

\section{Introduction}

At a time of unprecedented change in the Earth's climate [1-3], there is an unparalleled level of research into more sustainable and less damaging sources of energy, and our ability to harness them. Solar and wind energy are particularly promising, but both are intermittent, which is driving research in a wide variety of fields, including electrolysis [4-6], hydrogen production [7-9], and energy storage [10-12]. At present, the energy storage technology receiving the most attention is the battery [13-16], although it is clear there are questions regarding its lifecycle and sustainability [17-19]. Despite this, and regardless of how such issues are ultimately addressed, electrochemical devices continue to constitute a key part of the solution to the world's energy and climate problems, and a key part of all electrochemical devices is the electrode.

The characterisation of the physical connection between a solid electrode and a liquid electrolyte has been the subject of decades of research within the electrochemistry community. A well-designed electrode is able to

\footnotetext{
* Corresponding author

Email address: c.dunnill@swansea.ac.uk (Charles W Dunnill)
}

present an effective surface area that is many thousands of times larger than its geometric area, a measurement that is known as its Roughness Factor (RF) [20, 21]. Such a high level of porosity is key to the performance of many commonplace electrochemical devices, such as electrolysers [22, 23], fuel-cells [24] and batteries.

However, questions can arise about whether high surface area can only be achieved at the expense of reduced mass transport, due to reduced mobility within pores, with transmission lines being employed to model the behaviour of individual pores [25], and fractals to model the behaviour of whole electrodes [26]. Wherever there is reduced mobility, the effect of normal liquid viscosity is amplified significantly, such that convection and diffusion are greatly reduced. This can be used to advantage, for example in thermally insulating materials and clothing, but is very much an undesired phenomenon in electrochemical devices.

The RF of an electrode can be determined using CyclicVoltammetry (CV), wherein the current is measured whilst the electrode voltage is cycled through a small range (typically $100 \mathrm{mV}$ ) around open-circuit potential (OCP) [27]. This measurement is based on the accepted principle that 
the RF is proportional to the Electrochemical Surface Area (ECSA) of the electrode, which in turn is proportional to the double-layer capacitance $\left(C_{D L}\right)$, such that:

$$
\begin{aligned}
R F & =\frac{C_{D L}}{C_{S} A} \\
\text { where } \quad C_{S} & =40 \mu \mathrm{F} \mathrm{cm}{ }^{-2}
\end{aligned}
$$

and $A$ is the exposed area of the electrode in $\mathrm{cm}^{2} . C_{S}$ is the specific capacitance of a perfectly uniform electrode, which is accepted to have the above value in alkaline media [28].

The assumption that all current is used to charge and discharge $C_{D L}$ permits a value for it to be calculated. In practice, the waveforms measured vary significantly in shape, making the calculation less straightforward. For example, a single Raney Nickel electrode (with coating 'Raney 1' as previously described [29]) was successively characterised using CV before and after various procedures, with the results as presented in Figure 1. Inspection of the waveforms reveals that not only has usage significantly altered the capacitance, but also the shape of the waveforms.

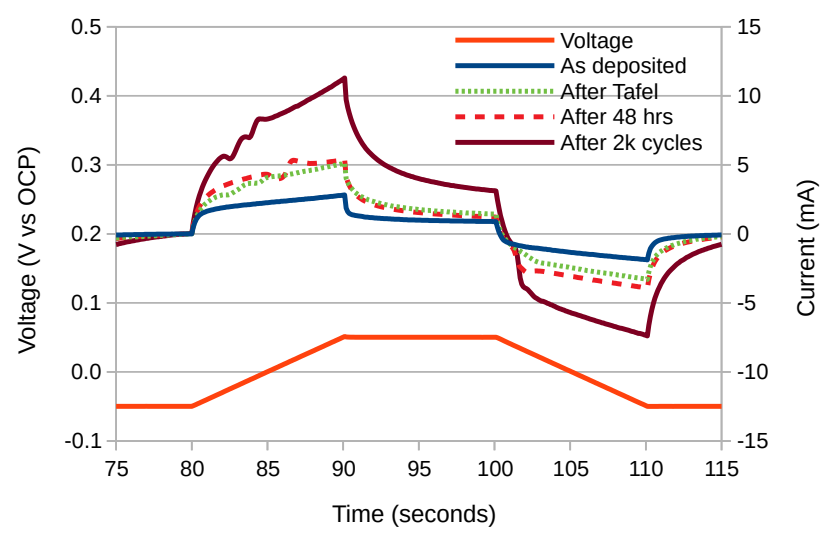

Figure 1: Cyclic Voltammetry waveforms measured for a single Raney Nickel electrode before and after various procedures connected with characterisation and ageing.

It is also clear that the waveforms feature ionisation peaks and troughs, and their presence would appear to preclude any calculation of equivalent circuit parameters at all. After all, it cannot be argued that all of the current is charging and discharging $C_{D L}$ if some of it is being used to alter the ionisation state of the electrode/electrolyte. In fact, the avoidance of such peaks is one of the primary objectives of performing measurements around OCP in the first place. However, because the voltammetry is cyclic, for every anodic peak there must be a (displaced) cathodic peak, and vice versa. Therefore, to at least a first order of approximation, an average may be taken of the anodic and cathodic best-fit parameters to generate values which cancel out their effect. In any case, the noise-rejection of the best-fit algorithm is such that convincing results are produced regardless, and highlights the fact that the method here presented is a shape-fitting algorithm, not a chargeintegration technique.

Electrochemical Impedance Spectroscopy (EIS) is a technique wherein the current is measured whilst a small oscillation of the electrode voltage around an a.c. operating point is swept through a range of frequencies. It is therefore an analogous technique to $\mathrm{CV}$, except that it is based in the frequency domain. It is typical to interpret the results of EIS by performing a best-fit against a Randles equivalent electrical circuit, as shown in Figure 2a [28, 3032]. Component $R_{S}$ represents the series resistance of the electrolyte between the working and reference electrodes and $C_{D L}$ represents the double-layer capacitance.

$R_{T}$ is normally taken to represent the transferresistance of any Faradaic chemical reaction that occurs between the electrode and the electrolyte. However, since the $\mathrm{CV}$ and EIS measurements are conducted around OCP, such reactions should either be absent or occurring at very low levels. In practice, $R_{T}$ can represent any process that involves discharge of the double-layer capacitance, and some value for it has been consistently observed in all experiments, therefore it is included. Note that if the value of $R_{T}$ is taken to infinity, then the response simplifies to that of an RC circuit, so this single equivalent circuit is able to emulate both RC and RCR networks. In Figure $2 \mathrm{~b}$ the equivalent circuit has been extended to an RCRCR network with the inclusion of two components labelled $R_{2}$ and $C_{2}$.

To improve the accuracy of the fit, the double-layer capacitance $C_{D L}$ can be replaced with a constant phase element (CPE). However, this raises the inverse problem of determining to how much capacitance a particular CPE corresponds. In the literature several different methods have been proposed, each based on specific assumptions, and each leading to different values [30, 33, 34]. This highlights the fundamental quandary at the heart of the CPE model, which is that although it is able to convincingly fit the frequency-domain response of many electrochemi- 


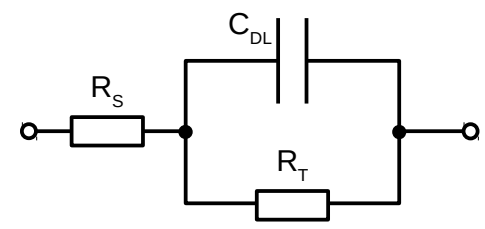

(a) Randles equivalent circuit

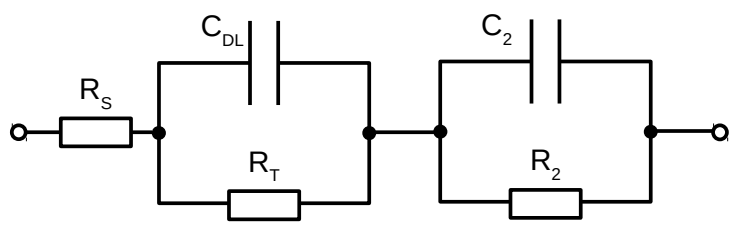

(b) extended Randles equivalent circuit

Figure 2: Simplified Randles electrical equivalent circuit as observed experimentally, and as extended to five components.

cal systems, its physical interpretation remains a matter for debate.

\section{Method}

This study is based on the results from 18 different working electrodes, as shown in Figure 5. The electrode coatings were TiN, Raney nickel and uncoated 316grade stainless-steel. All electrodes were mounted in a 3electrode cell constructed in a laminar fashion from lasercut acrylic $[35,36]$. The exposed area was $3 \mathrm{~cm} \times 3 \mathrm{~cm}$ for the working electrode, and $6 \mathrm{~cm} \times 6 \mathrm{~cm}$ for the 316 -grade stainless-steel counter electrode. Electrodes described as 'smooth' were used as delivered from sheet metal suppliers, and had not been polished in any way. The reference electrode was a commercial $\mathrm{Ag} / \mathrm{AgCl}$ design, which was routinely calibrated against a standard Calomel electrode. The electrolyte was $0.5 \mathrm{M} \mathrm{NaOH}$ at normal laboratory temperature, which was $20 \pm 1^{\circ} \mathrm{C}$.

Potentiostat. All electrochemical experiments were performed on an Ivium n-Stat potentiostat. All EIS results were analysed within the IviumSoft software package, wherein RCR and RQR equivalent circuits were fitted to the results (where $\mathrm{Q}$ is the symbol that represents a $\mathrm{CPE}$ ).

Cyclic Voltammetry. CV was performed within a $100 \mathrm{mV}$ range around OCP at rates of $10 \mathrm{mV} \mathrm{s}^{-1}$ or less. The potential was held for 10 seconds between changes of direction to allow diffusion gradients within the electrolyte to disperse. The results were imported into a bespoke website (https://fitting.gannon.me.uk) written in the PHP/MySQL languages, wherein a time-domain RCR equivalent circuit was fitted to the waveforms using standard parametric gradient descent. All interested parties are hereby invited to register with the website, and thereafter to make use of it in the analysis of their own results.

Electrochemical Impedance Spectroscopy. EIS was performed between $0.1 \mathrm{~Hz}$ and $10 \mathrm{kHz}$ around $\mathrm{OCP}$, starting at low frequency. Method: impedance; Technique: Constant E; Amplitude: $10 \mathrm{mV}$. The electrode was pretreated for 120 seconds at OCP to reduce initial transient currents.

CPE Transient Simulation. Calculation of the transient response of a $\mathrm{CPE}$ involves solving the inverse Laplace transform of the driving voltage waveform [37-40]. Alternatively, it is possible to use a convolution integral [41]. The procedure followed in this paper was as laid out in Section S6 of the SI, which uses a convolution integral together with numerical methods based on the Euler method. Together, these can simulate the transient response of a $\mathrm{CPE}$ to any arbitrary voltage waveform. The results for a voltage ramp were cross-checked using Mittag-Leffler functions, using the method laid out by C. Montella [38].

RCR Transient Best-fit. To perform a best-fit of the RCR Randles equivalent electrical circuit shown in Figure 2a to any given $\mathrm{CV}$ data, the time-domain response of the circuit to a voltage ramp must be understood. This can be calculated using Laplace transforms, with the analytical solution as presented in Equation 3.

$$
i(t)=\left[E t+F+G \exp \left(\frac{-t}{T}\right)\right] u(t)
$$

where $\quad E=\frac{\beta}{R_{S}+R_{T}}$

$$
\begin{aligned}
& F=\beta C_{D L}\left(\frac{R_{T}}{R_{S}+R_{T}}\right)^{2} \\
& G=-F \\
& T=\left(R_{S} \| R_{T}\right) C_{D L}
\end{aligned}
$$

where $\beta$ is the slope of the voltage ramp in $\mathrm{Vs}^{-1}$, and $u(t)$ is a unit step function at time $t=0$. It can thus be seen to be the sum of a ramp of magnitude $E$, a step of size $F$, and an exponential decay with time constant $T$. The derivation of the above analytical solution is as presented in Section S1 of the Supplementary Information (SI). 
If the values $E, F$ and $T$ are taken to be the axes of a three-dimensional solution space, then standard gradient descent techniques can be employed to find the position of the best-fit, i.e. the position that minimises a suitable cost-function, for example one defined as the square of the difference between the measured and analytical waveforms. Note that a fourth dimension is not required for parameter $G$, since it is equal to $-F$. From these parameters and the voltage ramp-rate $\beta$, the values of $R_{S}, R_{T}$ and $C_{D L}$ can be calculated using Equations 4 to 6 , with the derivation available in Section S2 of the SI.

$$
\begin{aligned}
C_{D L} & =\frac{(E T+F)^{2}}{\beta F} \\
R_{S} & =\frac{T}{F} \sqrt{\frac{\beta F}{C_{D L}}} \\
R_{T} & =\frac{1}{E} \sqrt{\frac{\beta F}{C_{D L}}}
\end{aligned}
$$

A best-fit between the analytical solution presented in Equation 3 and the time-domain current waveform measured during $\mathrm{CV}$ produces optimal derived values for $R_{S}$, $R_{T}$ and $C_{D L}$. This is based on the assumption that the electrode/electrolyte interface can indeed be modelled by the simplified Randles electrical equivalent circuit presented in Figure 2a, which is generally accepted [28, 30$32,42-44]$. There is at present no analytical solution for the fitting of an RQR network to $\mathrm{CV}$ results.

RCRCR Transient Best-fit. To perform a best-fit of the 5-component equivalent electrical circuit shown in Figure $2 \mathrm{~b}$, measurements from both the $\mathrm{CV}$ and EIS results were combined in order to generate enough accurate information. It should be noted that there are always two best-fit solutions, since the two RC networks can be interchanged without affecting the circuit's externally observed behaviour. The time-domain response of the circuit can be calculated using Laplace transforms, with the full analytical solution as presented in Equation 7.

$$
i(t)=\left[E t+F+G \exp \left(-\alpha_{1} t\right)+H \exp \left(-\alpha_{2} t\right)\right] u(t)
$$

$$
\text { where } \quad \begin{aligned}
E & =\frac{\beta \omega_{2} \omega_{3}}{\alpha_{1} \alpha_{2}} \\
F & =\frac{\beta\left(\omega_{2}+\omega_{3}\right)-E\left(\alpha_{1}+\alpha_{2}\right)}{\alpha_{1} \alpha_{2}} \\
G & =\frac{\beta-E-F \alpha_{2}}{\alpha_{2}-\alpha_{1}} \\
H & =-(F+G) \\
\alpha_{1} & =\frac{b-\sqrt{b^{2}-4 a c}}{2 a} \\
\alpha_{2} & =\frac{b+\sqrt{b^{2}-4 a c}}{2 a} \\
a & =R_{S} \\
\text { where } \quad b & =\left(R_{S}+R_{T}\right) \omega_{2}+\left(R_{S}+R_{2}\right) \omega_{3} \\
c & =\left(R_{S}+R_{T}+R_{2}\right) \omega_{2} \omega_{3} \\
\omega_{2} & =\frac{1}{R_{T} C_{D L}} \text { and } \omega_{3}=\frac{1}{R_{2} C_{2}}
\end{aligned}
$$

where $\beta$ is the slope of the voltage ramp in $\mathrm{Vs}^{-1}$, and $u(t)$ is a unit step function at time $t=0$. It can thus be seen to be the sum of a ramp of magnitude $E$, a step of size $F$, and two exponential decays. The full derivation of the above analytical solution is as presented in Section S3 of the SI. The value of $R_{S}$ can be determined directly from EIS, as can $\alpha_{2}$, and the values of $E, F$ and $T$ (where $T=1 / \alpha_{1}$ ) can be accurately measured from CV curve-fitting. This provides 5 measurable quantities, which together provide sufficient degrees of freedom to determine all 5-components of the equivalent circuit. Parameter $H$ can be visually estimated to further guide the descent if applicable.

Unfortunately, no reverse functions have been derived to generate the 5-component values directly from the measurable values. Nevertheless, it is still possible (given that $R_{S}$ is already known) to perform gradient descent within the four-dimensional solution space defined by the other components. Also, if the assumption is made that $R_{S}$ is much less than both $R_{T}$ and $R_{2}$, the above equations simplify greatly, and the solution space reduces to just twodimensions (see Section S4 of the SI).

Therefore, the method employed was to scan the twodimensional simplified solution space in order to generate a starting point for subsequent four-dimensional gradient descent. In this way, convergence onto one of the two bestfit solutions was reliably achieved. The method is outlined with an example in Section S5 of the SI. 


\section{Results}

The CV results for a smooth, TiN-coated 316-grade stainless steel electrode are as presented in Figure 3. Upon each change of direction, the current waveform is observed to consist of a curved RC-type response which decays to a ramp after several seconds. For this waveform, the RCR Transient Best-fit procedure was performed within a 5second window, as highlighted by the dashed grey rectangles. This ensured that approximately half the window contained a curved response, and half a linear response, a situation which was found to give the best trade-off between competing best-fit parameters. For other waveforms the window was chosen as appropriate.

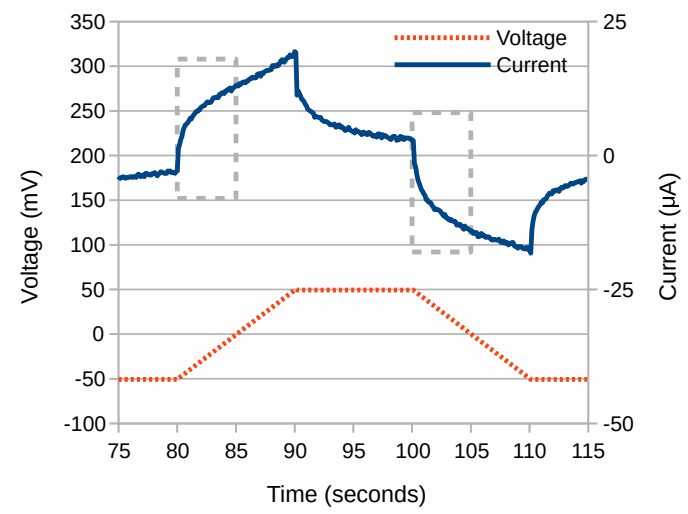

(a) Measured waveforms

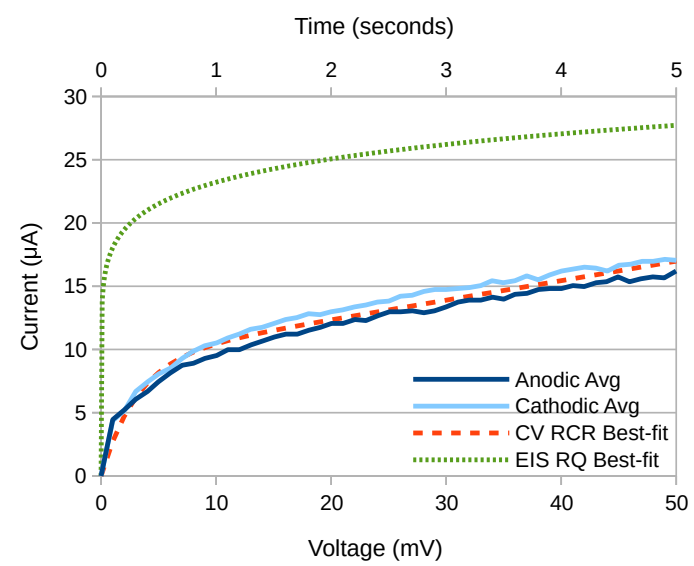

(b) Overlaid view of dashed rectangles

Figure 3: Cyclic Voltammetry results for a smooth, TiN-coated 316grade stainless-steel electrode at $10 \mathrm{mV} \mathrm{s}^{-1}$ showing (a) the unprocessed measurements and (b) the detail overlaid view of the dashed rectangles, where the current has been normalised to begin at $0 \mathrm{~A}$, together with the responses of the best-fit RCR and RQR networks.

The result of the RCR transient best-fit procedure for both rectangles in Figure $3 \mathrm{a}$ is as presented as the dashed orange line in Figure 3b. Note that the current has been normalised to begin at $0 \mathrm{~A}$, and that the cathodic waveform has been inverted so that it can be overlaid. The corresponding parametric values for each sweep are as presented in Table 1. The match between the observed response and that of an RCR network is quite close, which suggests that it is an appropriate equivalent circuit. However, the inferred series resistance $\left(R_{S}\right)$ of $320 \Omega$ is misleading, since no such resistance exists.

\begin{tabular}{|c|c|ccc|ccc|}
\hline Parameter & $\boldsymbol{\beta}$ & $\mathbf{E}$ & $\mathbf{F}$ & $\mathbf{T}$ & $\mathbf{R}_{\mathbf{S}}$ & $\mathbf{R}_{\mathbf{T}}$ & $\mathbf{C}_{\mathbf{D L}}$ \\
\hline Units & $\mathrm{mV} \mathrm{s}^{-1}$ & $\mathrm{\mu As}^{-1}$ & $\mu \mathrm{A}$ & $\mathrm{ms}$ & $\Omega$ & $\Omega$ & $\mu \mathrm{F}$ \\
\hline Anodic 1 & 10.0 & 1.57 & 8.76 & 275 & 299 & 6070 & 964 \\
\hline Cathodic 1 & 10.0 & 1.57 & 9.95 & 325 & 311 & 6060 & 1100 \\
\hline Anodic 2 & 10.0 & 1.54 & 8.48 & 295 & 330 & 6160 & 941 \\
\hline Cathodic 2 & 10.0 & 1.50 & 9.84 & 352 & 340 & 6330 & 1090 \\
\hline Average & & $\mathbf{1 . 5 5}$ & $\mathbf{9 . 2 6}$ & $\mathbf{3 1 2}$ & $\mathbf{3 2 0}$ & $\mathbf{6 1 5 0}$ & $\mathbf{1 0 2 0}$ \\
\hline
\end{tabular}

Table 1: Best-fit values of an RCR network to the observed waveforms in Figure 3.

The EIS measurements for the same electrode are as presented in Figure 4, with the best-fit RQR network parameters to these measurements (generated by IviumSoft) as presented in Table 2. Since the measurements were conducted around OCP, the resulting value of the transfer resistance $R_{T}$ is very high at $13 \mathrm{k} \Omega$. This is so high that it can be ignored, with the equivalent circuit thereby reducing to just an RQ network. Significantly, the $R_{S}$ value obtained from EIS is just $0.89 \Omega$, which is more than 350 times smaller than that obtained from CV curve-fitting.

\begin{tabular}{|c|c|c|c|c|}
\hline Parameter & $\mathbf{R}_{\mathbf{S}}$ & $\mathbf{Q}_{\mathbf{D L}}$ & $\boldsymbol{\alpha}$ & $\mathbf{R}_{\mathbf{T}}$ \\
\hline Units & $\Omega$ & $\mathrm{Ss}^{\alpha}$ & & $\mathrm{k} \Omega$ \\
\hline Value & 0.83 & 0.0022 & 0.89 & 13 \\
\hline
\end{tabular}

Table 2: Best-fit values of an RQR network to the observed waveforms in Figure 4.

The solid line on the Nyquist plot (Figure 4a) shows 'constant phase element' (CPE) behaviour, where at progressively lower frequencies the trace maintains a constant phase angle relative to the origin. At other values of bias voltage (away from OCP) this produces the classic 'flattened-semicircle' that is characteristic of many electrochemical systems $[32,45-50]$. The response of the RQ network is shown as the dotted green lines in Figure 4, and produces a very close match to the observed data.

By contrast, when the RQ network was simulated in the time domain to produce its transient response, the result is as shown as the dotted green line in Figure $3 \mathrm{~b}$. This produced a poor match, particularly within the first few 


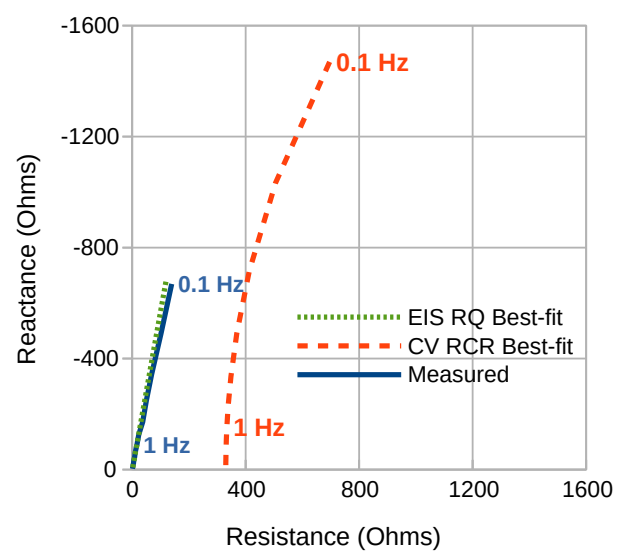

(a) Nyquist plot

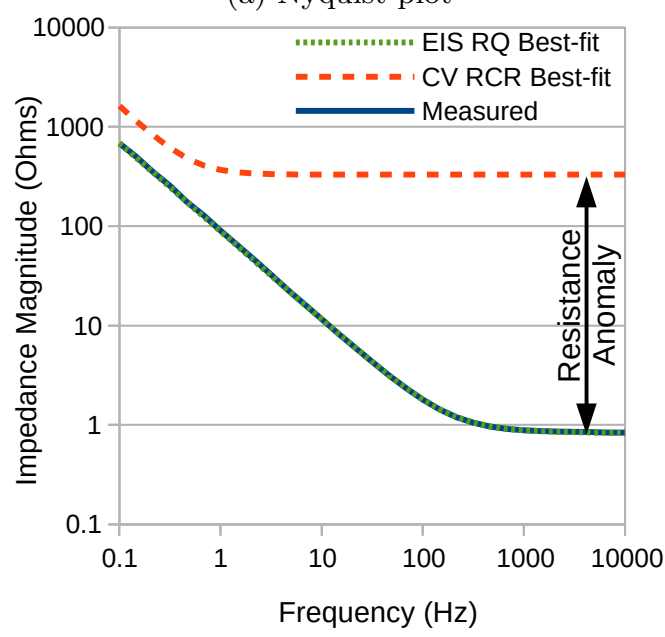

(b) Bode Magnitude plot

Figure 4: EIS measurements for the electrode presented in Figure 3, together with the responses of the best-fit RCR and RQR models.

milliseconds, where the response of the RQ network is almost vertical. This is not surprising, since the pseudo-timeconstant of the RQ network ( $R$ times $Q$ ) is just $1.8 \mathrm{~ms}$. However, thereafter the RQ network produced a much slower exponential decay that is very similar to the $\mathrm{CV}$ measurement.

By performing an RCR Transient best-fit on the segment of this waveform from $20 \mathrm{~ms}$ onwards, so as to exclude the vertical section, a time-constant of $299 \mathrm{~ms}$ was measured by curve-fitting. This is very similar to the value of $312 \mathrm{~ms}$ presented in Table 1. This curve-fitting also produced an apparent series resistance of $369 \Omega$, which is very similar to the value of $320 \Omega$ from Table 1 . It thus appears that a single RQ network is able to match the EIS and $\mathrm{CV}$ results, but it does so by presenting two different time-constants.

This size of the apparent resistance mismatch is more clearly illustrated by the frequency response of the $\mathrm{CV}$ RCR best-fit, which is presented as the dashed orange lines in Figure 4. The Nyquist plot shows that the RCR network is constrained to produce a semicircle, since it is comprised of ideal resistors and capacitors. The resistance mismatch appears at high frequencies on the Bode magnitude plot in Figure 5b (hereafter referred to as the 'Resistance Anomaly' or $R_{A}$ ), which amounts to more than two orders of magnitude.

The Resistance Anomaly has been consistently observed across a wide variety of electrodes, whether coated or uncoated, used or unused, smooth or high surface area and porous. Furthermore, its magnitude has been observed to vary in inverse proportion to the roughness factor, as presented in Figure 5.

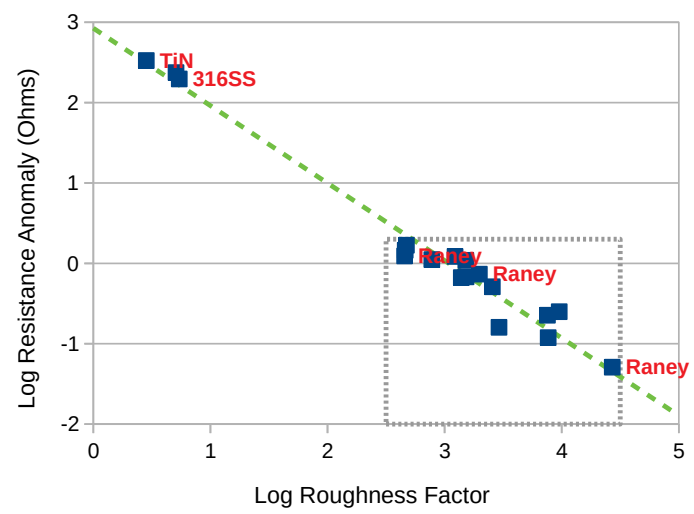

(a) Best-fit on log-log plot

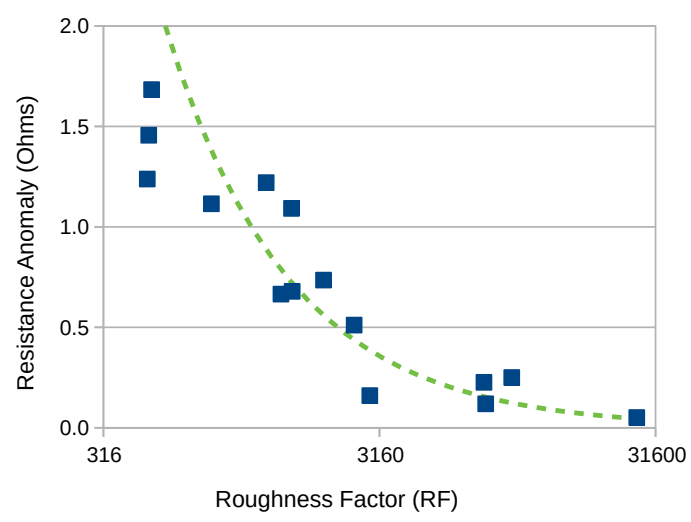

(b) Detail view of dotted rectangle on lin-log plot

Figure 5: Plot of Resistance Anomaly $\left(R_{A}\right)$ versus roughness factor $(\mathrm{RF})$ for various coated and uncoated electrodes.

The results show a direct relationship between $R_{A}$ and $\mathrm{RF}$ on a log-log plot that extends over 4 orders of magnitude. Figure 5b presents a detail view of the dotted box with one linear axis, to highlight how $R_{A}$ tends to zero as 
$\mathrm{RF}$ increases. The slope of the line in Figure $5 \mathrm{a}$ is -0.964 , which is close to -1 . This implies an inverse relationship, specifically:

$$
R_{A}=\frac{759 \mathrm{~m} \Omega \mathrm{m}^{2}}{A \cdot R F}
$$

where $759 \mathrm{~m} \Omega \mathrm{m}^{2}$ is an average figure generated across the complete set of electrodes. This figure will be independent of electrode area and the distance between working and reference electrodes.

Although the RQ network is able to match some of the electrode behaviour observed using $\mathrm{CV}$ in Figure $3 \mathrm{~b}$, it is hereby proposed that a better match could be achieved using a 5-component model. Inspection of the analytical solution of the 5-component RCRCR network, as presented in Equation 7, predicts that the time-domain response will be the sum of a ramp, a step, and two exponential decays. It may therefore be able to match the bi-exponential behaviour exhibited by the RQ network, whereby a rapid exponential decay is followed by a slower one.

Whilst Figure $3 \mathrm{~b}$ shows some evidence of this, it is much clearer in later waveforms recorded for the same electrode after a small amount of active gas evolution, as presented in Figures $6 \mathrm{a}$ and $6 \mathrm{~b}$. Note in the detail view that the current jumps quickly from 0 to $15 \mu \mathrm{A}$, and thereafter climbs more slowly, thereby indicating the presence of two time-constants.

Together with the EIS data presented in Figures $7 \mathrm{a}$ and $7 \mathrm{~b}$, the measurable parameters presented in Table 3 were extracted, where $R_{1}$ is the high frequency intercept from the Nyquist plot, $\alpha_{2}$ is the breakpoint from the Bode magnitude plot, and $E, F$ and $T$ are the current ramprate, step-size and time-constant from the $\mathrm{CV}$. The value of $H$ is an estimate and is included to assist descent.

\begin{tabular}{|c|cc|c|cccc|}
\hline Parameter & $\mathbf{R}_{\mathbf{1}}$ & $\boldsymbol{\alpha}_{\mathbf{2}}$ & $\boldsymbol{\beta}$ & $\mathbf{E}$ & $\mathbf{H}$ & $\mathbf{F}$ & $\mathbf{T}$ \\
\hline Units & $\Omega$ & $\mathrm{rad} \mathrm{s}^{-1}$ & $\mathrm{mV} \mathrm{s}^{-1}$ & $\mu_{\mathrm{A} \mathrm{s}}^{-1}$ & $\mu \mathrm{A}$ & $\mu \mathrm{A}$ & $\mathrm{ms}$ \\
\hline Anodic 1 & & & 13.3 & 2.11 & -15.0 & $7.45-H$ & 493 \\
\hline Cathodic 1 & & & 13.3 & 1.52 & -15.5 & $10.4-H$ & 561 \\
\hline Anodic 2 & & & 13.3 & 2.11 & -15.2 & $7.54-H$ & 483 \\
\hline Cathodic 2 & & & 13.3 & 1.49 & -15.7 & $10.4-H$ & 548 \\
\hline Average & $\mathbf{0 . 7 7 8}$ & $\mathbf{1 4 9 4}$ & $\mathbf{1 3 . 3}$ & $\mathbf{1 . 8 1}$ & $\mathbf{- 1 5 . 3}$ & $\mathbf{2 4 . 3}$ & $\mathbf{5 2 1}$ \\
\hline
\end{tabular}

Table 3: Measurable parameters determined from the data presented in Figures 6 and 7.

From these measurable parameters, four-dimensional gradient descent was performed to determine all of the values for the 5-component RCRCR network equivalent circuit, which are as presented in column 'Electrode 1' of

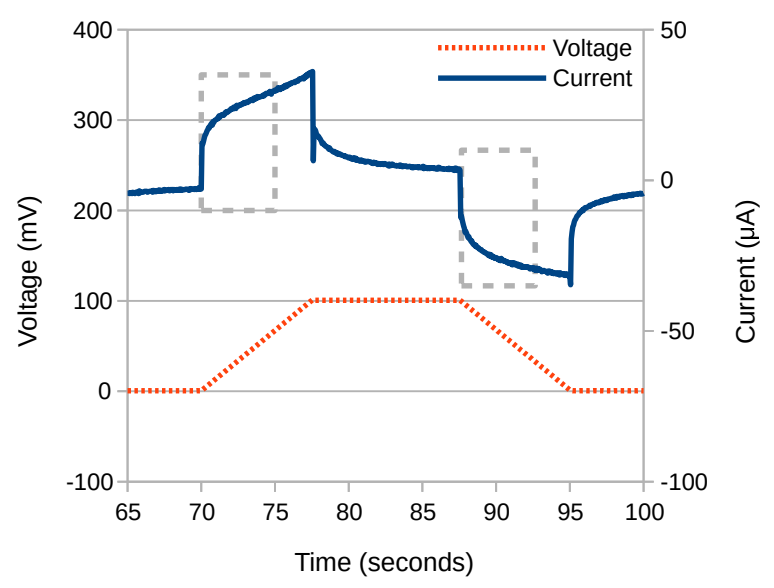

(a) $\mathrm{CV}$

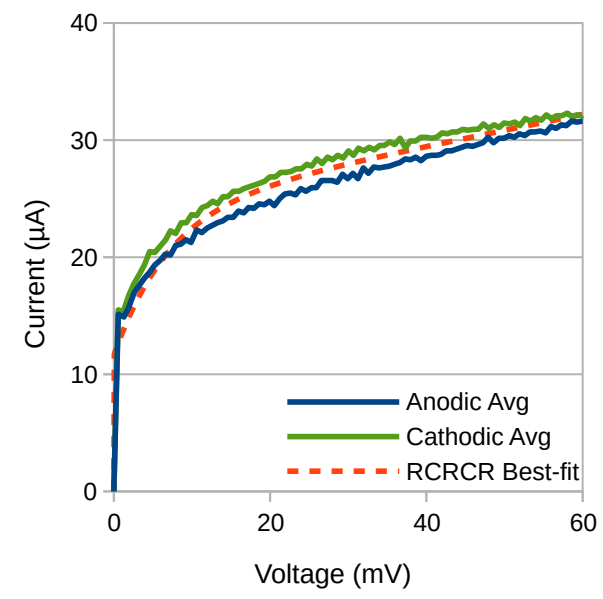

(b) CV Detail View

Figure 6: CV measurements obtained for the TiN-coated 316SS electrode in Figure 3 after active gas evolution. Figure (a) presents the $\mathrm{CV}$ data as recorded, and Figure (b) presents overlaid voltammograms of the dashed grey boxes. EIS bias voltage: $0 \mathrm{~V}$; CV sweep rate: $13.3 \mathrm{mV} \mathrm{s}^{-1}$; Electrolyte: $0.5 \mathrm{M} \mathrm{NaOH}$ at laboratory temperature.

Table 4. The time-domain response of this network is included as the dashed orange line in Figure 6, and the frequency domain response in Figure 7 . From these it can be seen that the equivalent circuit is now accurately modelling the measured behaviour of the electrode.

The table also includes the RCRCR best-fit parameters for two other electrodes with widely varying Roughness Factors. A progression can be seen across the table, which can be more easily visualised in Figure 8a. From this figure it can be seen that the values of $R_{T}, C_{D L}, R_{2}$ and $C_{2}$ are linearly related, which implies that they are not independent phenomena. To investigate this, a CV experiment was repeated with and without vigorous pumped 


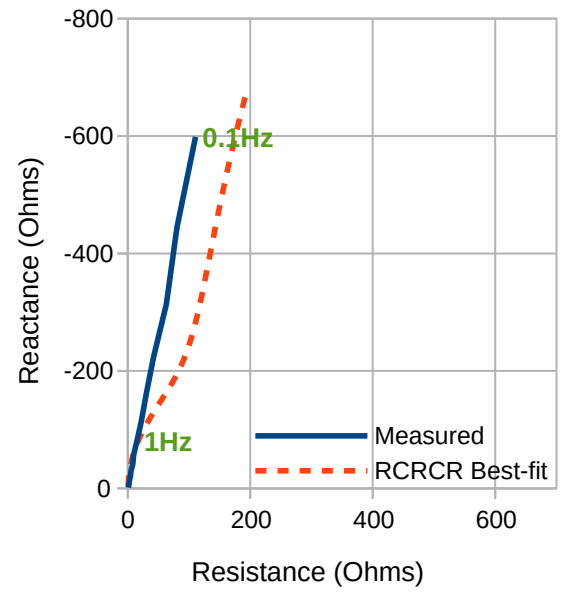

(a) EIS Nyquist Plot

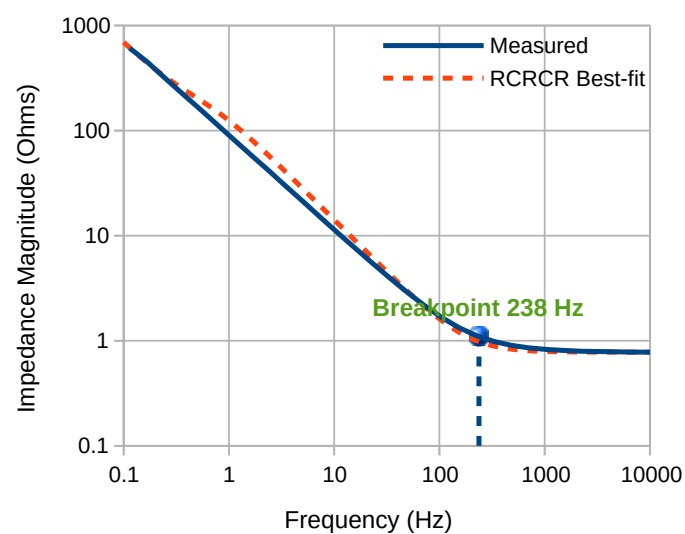

(b) EIS Bode Magnitude

Figure 7: EIS measurements obtained for the TiN-coated 316SS electrode in Figure 3 as (a) a Nyquist plot and (b) a Bode magnitude plot, from which the breakpoint frequency can be determined. The response of the best-fit RCRCR network to these data is included as a dashed orange line.

\begin{tabular}{|c|l|l|l|}
\hline Parameter & Electrode 1 & Electrode 2 & Electrode 3 \\
\hline $\mathbf{R F}$ & 2.8 & 472 & 7556 \\
\hline $\mathbf{R}_{\mathbf{S}}$ & $0.778 \Omega$ & $0.913 \Omega$ & $0.946 \Omega$ \\
\hline $\mathbf{R}_{\mathbf{T}}$ & $5610 \Omega$ & $101 \Omega$ & $4.53 \Omega$ \\
\hline $\mathbf{C}_{\mathbf{D L}}$ & $2420 \mu \mathrm{F}$ & $185 \mathrm{mF}$ & $2.96 \mathrm{~F}$ \\
\hline $\mathbf{R}_{\mathbf{2}}$ & $118 \Omega$ & $0.803 \Omega$ & $0.134 \Omega$ \\
\hline $\mathbf{C}_{\mathbf{2}}$ & $2100 \mu \mathrm{F}$ & $232 \mathrm{mF}$ & $4.56 \mathrm{~F}$ \\
\hline
\end{tabular}

Table 4: Best-fit values obtained for the 5-element equivalent circuit model matching the measurement data presented in Figures 6 and 7 (Electrode 1), as well as two other electrodes with much larger roughness factors.

circulation of the electrolyte, with the results as presented in Figure 8b. The results confirm that the measurements are unaffected by pumped circulation, and therefore do not arise as a result of bulk movements of the electrolyte, such as diffusion.

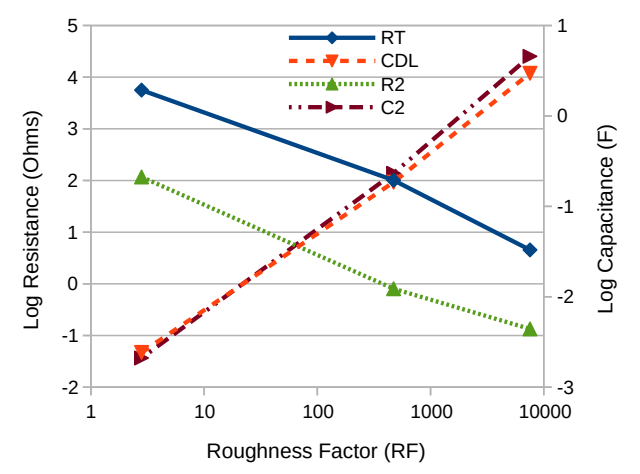

(a) Trends versus $\mathrm{RF}$

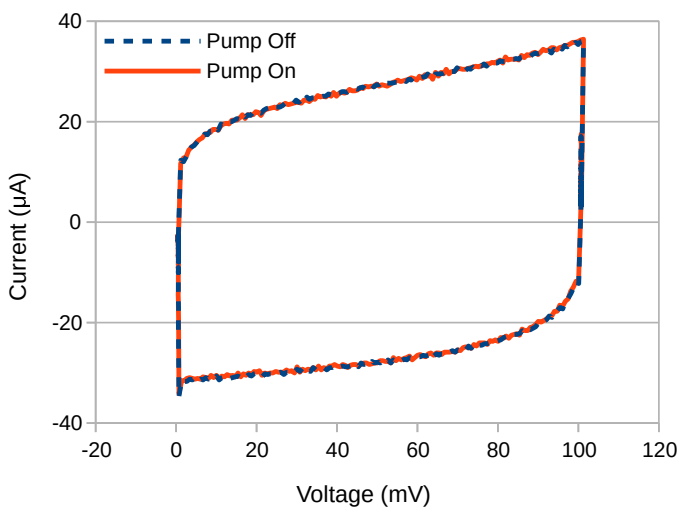

(b) Effect of pumped circulation

Figure 8: a) Trends in the RCRCR best-fit parameters as a function of roughness factor for the three electrodes presented in Table 4. b) Cyclic voltammograms of the TiN electrode with and without vigorous pumped circulation of the electrolyte. The pumping has no discernible effect.

\subsection{Computer Simulations}

Simulations were conducted to determine how the response of an RQ network to a voltage ramp varies with component values. The value of the apparent time-constant as a function of the resistance $R$ was measured using $R C R$ Transient Best-fit, with the results presented in Figure 9a.

The results show that the apparent time-constant of the RQ network remains invariant until the pseudo-timeconstant of the RQ network (i.e. $R$ times $Q$ ) approaches about $0.1 \mathrm{~s}$. A brief reduction is observed in the apparent time-constant, which is an artefact of the curve-fitting procedure as the difference between the two time-constants reduces. Thereafter, the RQ network exhibits just a single time-constant, which is dominated by the conventional product of resistance times capacitance, which is to be expected of an RC network. Since the shape of the transient response of the RQ network is governed by the pseudo-time-constant, the results for holding $R$ constant 


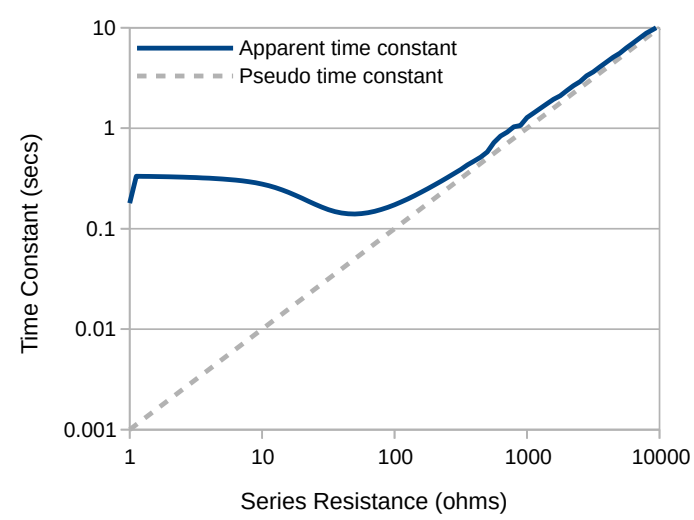

(a) Variation with $R$

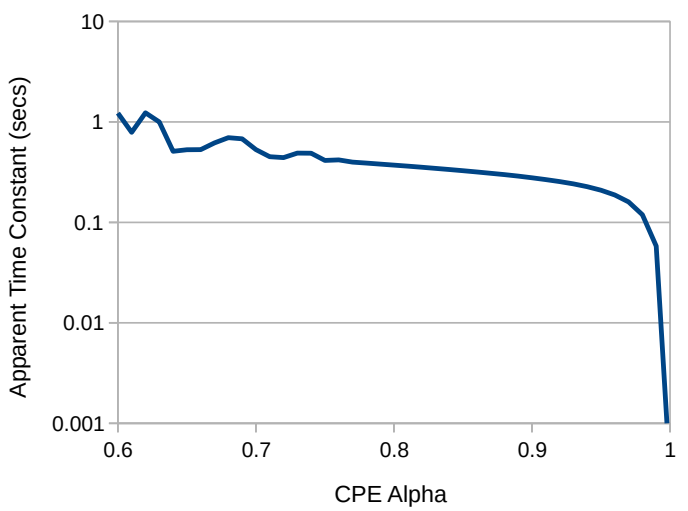

(b) Variation with $\alpha$

Figure 9: Apparent observed time constant of the RQ network versus component values. Segment start $=0.03 \mathrm{~s}$, finish $=5 \mathrm{~s} \mathrm{a)} Q=0.001$, $\alpha=0.9$ b) $R=1, Q=0.01$

and sweeping $Q$ are identical.

The results for sweeping the value of $\alpha$ are as presented in Figure 9b. These show that the time-constant is low for a perfect capacitor $(\alpha=1)$, but increases rapidly and is then relatively constant over a wide region. This region covers the typical values of $\alpha$ that occur in many electrochemical experiments, and certainly all of the results published in this paper. This means that if the first time-constant is short enough that a cyclicvoltammetry experiment fails to reveal it (for example as in Figure 3b), then only the second, longer time-constant will be observed. The shape of this curve is not determined by resistance at all, but entirely by the constant phase element.

\section{Conclusions}

This paper presents an accurate and reliable method for the fitting of a three-component RCR network to the measured response of an electrode during cyclicvoltammetry $(\mathrm{CV})$. This method employs the analytical derivation of the time-domain response of an RCR network to a voltage ramp, calculated using Laplace transforms. Three-dimensional gradient descent is then used to obtain the best-fit solution.

The frequency response of the same electrode, measured using electrochemical impedance spectroscopy (EIS), was fitted to an RQR network using conventional electrochemistry software, where Q represents a constant phase element (CPE). Since all measurements were conducted around OCP, the value of the transfer resistance was large and could be discounted, thereby simplifying to an RQ network.

Comparison of the best-fit RCR and RQ networks revealed an apparent resistance anomaly, which could amount to several hundred ohms (Figure 4b). It was observed across a wide variety of electrodes that the resistance anomaly was inversely proportional to the roughness factor $(\mathrm{RF})$ of the electrode, where the RF was proportional to the double-layer capacitance.

A simplified method was developed to simulate the transient-response of an RQ network to a voltage ramp, with example code presented in the PHP programming language. Inspection of this response reveals that a single RQ network is able to exhibit bi-exponential behaviour, with two separate time-constants (Figures $3 \mathrm{~b}$ and $6 \mathrm{~b}$ ). Such behaviour was also observed experimentally (Figure 6a), although not consistently.

This observation is therefore able to explain the apparent existence of the resistance anomaly, which arises because in practice the RCR gradient descent method measures the second time-constant of the RQ network. As shown by computer simulation (Figure 9a) this second time-constant is invariant for low values of the pseudotime-constant (those below $0.1 \mathrm{~s}$ ), which is typical in electrochemical cells containing normal high-conductivity electrolyte. Since $C$ is known to vary, but the time-constant ( $R$ times $C$ ) is fixed, the illusion is thus created that $R$ is inversely proportional to $C$ (Figure $5 \mathrm{~b}$ ).

A five-component RCRCR model was then proposed, with a fitting procedure based on measurable quantities derived from both the time-domain and frequency-domain measurements. This method employs the analytical derivation of the time-domain response of an RCRCR network 
to a voltage ramp, calculated using Laplace transforms. The method then makes uses of four-dimensional gradient descent to obtain the best-fit solution, with a mathematical simplification and a two-dimensional plot providing the starting point.

This five-component network produces the most accurate fit to the observed results, even though it does not contain a constant phase element. One of the component values is fixed and equal to the solution resistance, and the values of the other four are observed to vary linearly with electrode roughness factor (Figure 8a). This suggests that the fitting procedure is not revealing any new information about the electrode/electrolyte interface. This is because any constant phase element (such as the double-layer capacitance) can be expanded into an infinite series of parallel RC networks, where the component values of each RC network are a fixed percentage of the previous [51]. The RCRCR network can thus be regarded as the first expansion of an RQ network into an infinite $R(C R)$ network.

It would be interesting to extend the RCR transient curve-fitting technique to equivalent circuits containing more complex elements. These would include not just constant phase elements, but also inductors, finite and infinite Warburg impedances, and Gerischer. It would also be interesting to extend the technique to perform transient curve-fitting at voltages well away from OCP.

The most surprising and unexpected finding is that a single RQ network is able to exhibit bi-exponential behaviour, based on two time-constants. One of these is proportional to the product of $R$ and $Q$ (the pseudo-timeconstant), but the other is fixed at approximately $0.3 \mathrm{~s}$. Within limits, this time-constant is not a function of any of the three component values of the network i.e. $R, Q$ or $\alpha$, but emerges directly from the mathematical derivation (Figure S7 in the SI). It therefore appears to be an invariant property of the constant phase element itself, and one that has been consistently verified by experimental observation.

Funding. PhD student William Gannon, funded by a University of Swansea Zienkiewicz Scholarship.

Conflicts of Interest. The authors declare no conflict of interest.

\section{References}

[1] J. Bendell, "Deep Adaptation: a map for navigating climate tragedy," Water, 2016.

[2] W. Steffen, J. Rockström, K. Richardson, T. M. Lenton, C. Folke, D. Liverman, C. P. Summerhayes, A. D. Barnosky, S. E. Cornell, M. Crucifix, J. F. Donges, I. Fetzer, S. J. Lade, M. Scheffer, R. Winkelmann, and H. J. Schellnhuber, "Trajectories of the Earth System in the Anthropocene," Proceedings of the National Academy of Sciences, vol. 115, no. 33, pp. 82528259, 2018.

[3] EASAC, "Extreme weather events in Europe. Preparing for climate change adaptation: an update on EASAC's 2013 study," Tech. Rep. March, EASAC, 2018.

[4] R. Phillips and C. W. Dunnill, "Zero gap alkaline electrolysis cell design for renewable energy storage as hydrogen gas," $R S C$ Advances, vol. 6, no. 102, pp. 100643-100651, 2016.

[5] R. Phillips, A. Edwards, B. Rome, D. R. Jones, and C. W. Dunnill, "Minimising the ohmic resistance of an alkaline electrolysis cell through effective cell design," International Journal of Hydrogen Energy, vol. 42, no. 38, pp. 23986-23994, 2017.

[6] S. A. Grigoriev, V. N. Fateev, D. G. Bessarabov, and P. Millet, "Current status, research trends, and challenges in water electrolysis science and technology," International Journal of Hydrogen Energy, no. xxxx, 2020.

[7] A. Ursúa, I. San Martín, E. L. Barrios, and P. Sanchis, "Standalone operation of an alkaline water electrolyser fed by wind and photovoltaic systems," International Journal of Hydrogen Energy, vol. 38, no. 35, pp. 14952-14967, 2013.

[8] I. Dincer and C. Acar, "Review and evaluation of hydrogen production methods for better sustainability," International Journal of Hydrogen Energy, vol. 40, no. 34, pp. 11094-11111, 2014.

[9] F. Dawood, M. Anda, and G. M. Shafiullah, "Hydrogen production for energy: An overview," International Journal of Hydrogen Energy, vol. 45, no. 7, pp. 3847-3869, 2020.

[10] T. R. Cook, D. K. Dogutan, S. Y. Reece, Y. Surendranath, T. S. Teets, and D. G. Nocera, "Solar Energy Supply and Storage for the Legacy and Nonlegacy Worlds," Chemical Reviews, vol. 110, pp. 6474-6502, 2010.

[11] D. Jones, R. Phillips, W. Gannon, B. Rome, M. Warwick, and C. Dunnill, "Photocapacitive CdS/WOx nanostructures for solar energy storage," Scientific Reports, vol. 9, no. 1, 2019.

[12] J. J. Brey, "Use of hydrogen as a seasonal energy storage system to manage renewable power deployment in Spain by 2030," International Journal of Hydrogen Energy, no. xxxx, 2020.

[13] D. Larcher and J. M. Tarascon, "Towards greener and more sustainable batteries for electrical energy storage," Nature Chemistry, vol. 7, no. 1, pp. 19-29, 2015.

[14] H. C. Hesse, M. Schimpe, D. Kucevic, and A. Jossen, Lithiumion battery storage for the grid - A review of stationary battery storage system design tailored for applications in modern power grids, vol. 10. MDPI, 2017.

[15] V. Papadopoulos, J. Desmet, J. Knockaert, and C. Develder, "Improving the utilization factor of a PEM electrolyzer powered by a 15 MW PV park by combining wind power and battery storage - Feasibility study," International Journal of Hydrogen Energy, vol. 43, no. 34, pp. 16468-16478, 2018.

[16] Z. Yuan, W. Wang, H. Wang, and A. Yıldızbas, "Allocation 
and sizing of battery energy storage system for primary frequency control based on bio- inspired methods : A case study," no. $\operatorname{xxxx}, 2020$.

[17] J. J. Hwang, J. K. Kuo, W. Wu, W. R. Chang, C. H. Lin, and S. E. Wang, "Lifecycle performance assessment of fuel cel1/battery electric vehicles," International Journal of Hydrogen Energy, vol. 38, no. 8, pp. 3433-3446, 2013.

[18] M. Romare and L. Dahllöf, "The Life Cycle Energy Consumption and Greenhouse Gas Emissions from Lithium-Ion Batteries," Tech. Rep. C, IVL Swedish Environmental Research Institute, 2017.

[19] C. Buchal, H.-d. Karl, and H.-w. Sinn, "Kohlemotoren , Windmotoren und Dieselmotoren : Was zeigt die CO2-Bilanz?," vol. 25, no. 72, pp. 40-54, 2019.

[20] V. Ganesh and V. Lakshminarayanan, "Preparation of high surface area nickel electrodeposit using a liquid crystal template technique," Electrochimica Acta, vol. 49, no. 21, pp. 3561-3572, 2004.

[21] R. Solmaz, "Gold-supported activated NiZn coatings: hydrogen evolution and corrosion studies," International Journal of Energy Research, vol. 41, pp. 1452-1459, 2017.

[22] J. Li, X. Li, Y. Luo, Q. Cen, Q. Ye, X. Xu, and F. Wang, "Cobalt carbonate hydroxide mesostructure with high surface area for enhanced electrocatalytic oxygen evolution," International Journal of Hydrogen Energy, vol. 43, no. 20, pp. 96359643, 2018.

[23] X. Zhang, J. Hampshire, K. Cooke, X. Li, D. Pletcher, S. Wright, and K. Hyde, "High surface area coatings for hydrogen evolution cathodes prepared by magnetron sputtering," International Journal of Hydrogen Energy, vol. 40, no. 6, pp. 2452-2459, 2015.

[24] P. Hong, L. Xu, J. Li, and M. Ouyang, "Modeling and analysis of internal water transfer behavior of PEM fuel cell of large surface area," International Journal of Hydrogen Energy, vol. 42, no. 29, pp. 18540-18550, 2017.

[25] J. Bisquert, "Theory of the impedance of electron diffusion and recombination in a thin layer," Journal of Physical Chemistry B, vol. 106, no. 2, pp. 325-333, 2002.

[26] R. Kant, "General theory of arbitrary potential sweep methods on an arbitrary topography electrode and its application to random surface roughness," Journal of Physical Chemistry C, vol. 114, no. 24, pp. 10894-10900, 2010.

[27] C. C. L. McCrory, S. Jung, I. M. Ferrer, S. M. Chatman, J. C. Peters, and T. F. Jaramillo, "Benchmarking Hydrogen Evolving Reaction and Oxygen Evolving Reaction Electrocatalysts for Solar Water Splitting Devices," Journal of the American Chemical Society, vol. 137, no. 13, pp. 4347-4357, 2015.

[28] C. C. L. McCrory, S. Jung, J. C. Peters, and T. F. Jaramillo, "Benchmarking heterogeneous electrocatalysts for the oxygen evolution reaction," Journal of the American Chemical Society, vol. 135, no. 45, pp. 16977-16987, 2013.

[29] W. Gannon and C. Dunnill, "Raney Nickel 2.0: Development of a high-performance bifunctional electrocatalyst," Electrochimica Acta, vol. 322, p. 134687, 2019.

[30] G. J. Brug, A. L. G. van den Eeden, M. Sluyters-Rehbach, and J. H. Sluyters, "The analysis of electrode impedances complicated by the presence of a constant phase element," Journal of Electroanalytical Chemistry, vol. 176, no. 1-2, pp. 275-295, 1984.

[31] I. Herraiz-Cardona, E. Ortega, L. Vázquez-Gómez, and V. Pérez-Herranz, "Double-template fabrication of threedimensional porous nickel electrodes for hydrogen evolution reaction," International Journal of Hydrogen Energy, vol. 37, no. 3, pp. 2147-2156, 2012.

[32] C. González-Buch, I. Herraiz-Cardona, E. Ortega, J. GarcíaAntón, and V. Pérez-Herranz, "Synthesis and characterization of macroporous $\mathrm{Ni}$, Co and Ni-Co electrocatalytic deposits for hydrogen evolution reaction in alkaline media," International Journal of Hydrogen Energy, vol. 38, no. 25, pp. 10157-10169, 2013.

[33] C. H. Hsu and F. Mansfeld, "Technical Note : Concerning the Conversion of the Constant Phase Element Parameter Yo into a Capacitance," Corrosion, vol. 57, no. 9, pp. 747-748, 2001.

[34] V. Jovic, "Determination of the correct value of Cdl from the impedance results fitted by the commercially available software," tech. rep., University of Belgrade, Belgrade, 2003.

[35] G. Passas and C. W. Dunnill, "Water Splitting Test Cell for Renewable Energy Storage as Hydrogen Gas," Fundamentals of Renewable Energy and Applications, vol. 5, no. 5, pp. 3-8, 2015 .

[36] W. W. J. Gannon, D. R. D. Jones, and C. C. W. Dunnill, "Enhanced Lifetime Cathode for Alkaline Electrolysis Using Standard Commercial Titanium Nitride Coatings," Processes, vol. 7, no. 2, p. 112, 2019.

[37] M. T. Ehrensberger and J. L. Gilbert, "A time-based potential step analysis of electrochemical impedance incorporating a constant phase element: A study of commercially pure titanium in phosphate buffered saline," Journal of Biomedical Materials Research - Part A, vol. 93, no. 2, pp. 576-584, 2010.

[38] C. Montella, "LSV/CV modelling of electrochemical reactions with interfacial CPE behaviour, using the generalised Mittag-Leffler function," Journal of Electroanalytical Chemistry, vol. 667, pp. 38-47, 2012.

[39] C. S. Cheng, H. S. H. Chung, R. W. H. Lau, and K. Y. W. Hong, "Time-Domain Modeling of Constant Phase Elements for Simulation of Lithium Battery Behavior," IEEE Transactions on Power Electronics, vol. 34, no. 8, pp. 7573-7587, 2019.

[40] E. Locorotondo, L. Pugi, L. Berzi, M. Pierini, S. Scavuzzo, A. Ferraris, A. G. Airale, and M. Carello, "Modeling and simulation of Constant Phase Element for battery Electrochemical Impedance Spectroscopy," 5th International Forum on Research and Technologies for Society and Industry: Innovation to Shape the Future, RTSI 2019 - Proceedings, pp. 225-230, 2019.

[41] V. Athanasiou and Z. Konkoli, "On the efficient simulation of electrical circuits with constant phase elements: The Warburg element as a test case," International Journal of Circuit Theory and Applications, vol. 46, no. 5, pp. 1072-1090, 2018.

[42] M. D. Levi and D. Aurbach, "Simultaneous Measurements and Modeling of the Electrochemical Impedance and the Cyclic Voltammetric Characteristics of Graphite Electrodes Doped with Lithium," The Journal of Physical Chemistry B, vol. 101, no. 23, pp. 4630-4640, 2002.

[43] M. P. Marceta Kaninski, V. M. Nikolic, G. S. Tasic, and 
Z. L. Rakocevic, "Electrocatalytic activation of Ni electrode for hydrogen production by electrodeposition of Co and $\mathrm{V}$ species," International Journal of Hydrogen Energy, vol. 34, no. 2, pp. 703-709, 2009.

[44] I. Herraiz-Cardona, E. Ortega, and V. Pérez-Herranz, "Impedance study of hydrogen evolution on $\mathrm{Ni} / \mathrm{Zn}$ and $\mathrm{Ni}$ $\mathrm{Co} / \mathrm{Zn}$ stainless steel based electrodeposits," Electrochimica Acta, vol. 56, no. 3, pp. 1308-1315, 2011.

[45] N. V. Krstajić, V. D. Jović, L. Gajić-Krstajić, B. M. Jović, A. L. Antozzi, and G. N. Martelli, "Electrodeposition of Ni-Mo alloy coatings and their characterization as cathodes for hydrogen evolution in sodium hydroxide solution," International Journal of Hydrogen Energy, vol. 33, no. 14, pp. 3676-3687, 2008.

[46] T. G. Douglas, A. Cruden, and D. Infield, "Development of an ambient temperature alkaline electrolyser for dynamic operation with renewable energy sources," International Journal of Hydrogen Energy, vol. 38, no. 2, pp. 723-739, 2013.

[47] A. Tozar and I. H. Karahan, "Structural and corrosion protection properties of electrochemically deposited nano-sized Zn-Ni alloy coatings," Applied Surface Science, vol. 318, pp. 15-23, 2014.

[48] Z. Huang, Z. Chen, Z. Chen, C. Lv, H. Meng, and C. Zhang, "Ni12P5 nanoparticles as an efficient catalyst for hydrogen generation via electrolysis and photoelectrolysis," ACS Nano, vol. 8, no. 8, pp. 8121-8129, 2014.

[49] R. Solmaz, A. Salcı, H. Yüksel, M. Doğrubaş, and G. Kardaş, "Preparation and characterization of Pd-modified Raney-type NiZn coatings and their application for alkaline water electrolysis," International Journal of Hydrogen Energy, vol. 42, no. 4, pp. 2464-2475, 2017.

[50] T. Yang, J. Liu, H. Finklea, S. Lee, W. K. Epting, R. Mahbub, T. Hsu, P. A. Salvador, H. W. Abernathy, and G. A. Hackett, "An efficient approach for prediction of Warburg-type resistance under working currents," International Journal of Hydrogen Energy, vol. 43, no. 32, pp. 15445-15456, 2018.

[51] J. Valsa and J. Vlach, "RC models of a constant phase element," International Journal of Circuit Theory and Applications, vol. 41, no. 1, pp. 59-67, 2013. 


\section{Supplementary Information}

This document contains the Supplementary Information for the manuscript "Apparent disagreement between cyclic voltammetry and electrochemical impedance spectroscopy explained by time-domain simulation of constant phase elements" by Gannon, W.J.F. et al.

(Note: All figures, equations, tables and sections in the Supplementary Information are prefixed with the character 'S'. If no ' $\mathrm{S}$ ' is present, this means that the link refers to the main manuscript.)

\section{S1. RCR Network: Derivation of response to a voltage ramp.}

The time-domain response can be calculated using Laplace transforms, wherein active components such as capacitors are replaced by their s-domain equivalent circuit, as shown in Figures S1a and S1b.

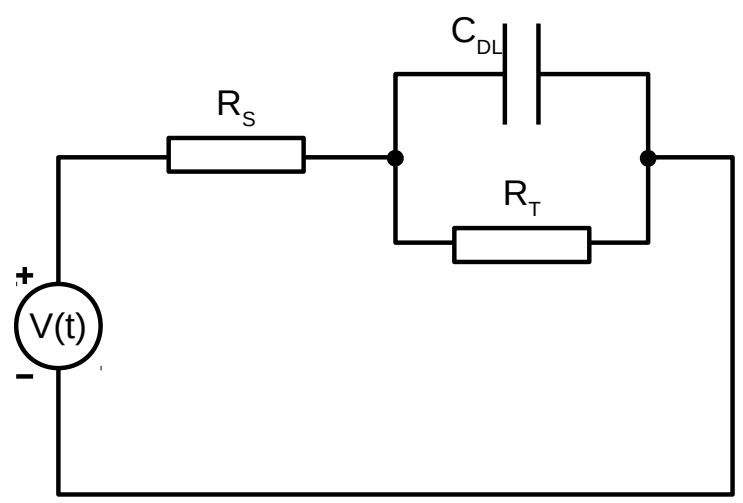

(a) The simplified Randles electrical equivalent circuit

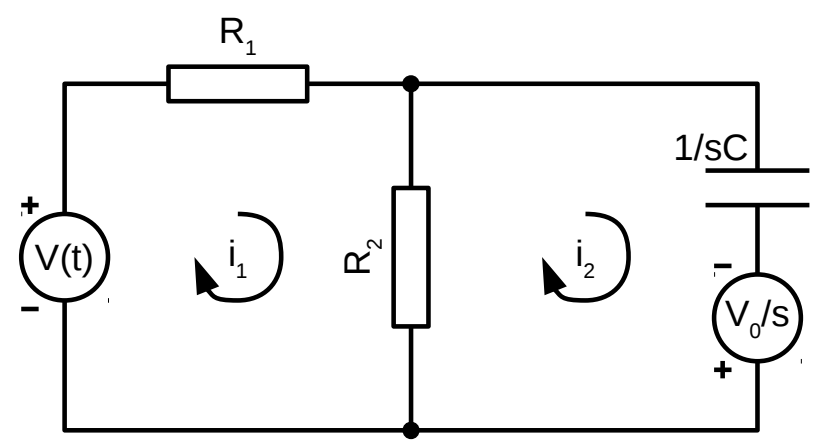

(b) As redrawn for mesh current analysis in s-domain

Figure S1: Equivalent circuit for the electrode/electrolyte interface extended to include transfer resistance $R_{T}$

The result of performing mesh-current analysis on the rearranged circuit is the following two equations:

$$
\begin{aligned}
R_{1} i_{1}+R_{2}\left(i_{1}-i_{2}\right) & =V(t) \\
\frac{i_{2}}{s C}+R_{2}\left(i_{2}-i_{1}\right) & =\frac{V_{0}}{s}
\end{aligned}
$$

From Table $\mathrm{S} 1$ and since the function of the driving voltage $V(t)$ is a ramp, this can be expressed in the s-domain as:

$$
V(s)=\frac{\beta}{s^{2}}
$$

where $\beta$ is the ramp-rate expressed in volts per second. Assuming that the charge on the capacitor at time $t=0$ is $0 \mathrm{~V}$, which means that $V_{0}=0$, Equation $\mathrm{S} 2$ can be rewritten as:

$$
\begin{aligned}
i_{2}\left(\frac{1}{s C}+R_{2}\right)-R_{2} i_{1} & =0 \\
\text { therefore } \quad i_{2} & =\left(\frac{s}{s+\frac{1}{R_{2} C}}\right) i_{1}
\end{aligned}
$$


Substituting Equation S5 in Equation S1 produces:

$$
\begin{gathered}
\left(R_{1}+R_{2}\right) i_{1}-R_{2}\left(\frac{s}{s+\frac{1}{R_{2} C}}\right) i_{1}=\frac{\beta}{s^{2}} \\
{\left[\frac{\left(R_{1}+R_{2}\right)\left(s+\frac{1}{R_{2} C}\right)-s R_{2}}{\left(s+\frac{1}{R_{2} C}\right)}\right] i_{1}=\frac{\beta}{s^{2}}}
\end{gathered}
$$

Dividing both sides by $R_{1}$ and rearranging produces:

$$
\begin{aligned}
\left(s+\frac{1}{R_{2} C}+\frac{1}{R_{1} C}\right) i_{1} & =\frac{\beta}{R_{1}}\left(\frac{s+\frac{1}{R_{2} C}}{s^{2}}\right) \\
\text { therefore } i_{1}(s) & =\frac{\beta}{R_{1}}\left[\frac{s+\frac{1}{R_{2} C}}{s^{2}\left(s+\frac{1}{R_{12} C}\right)}\right]
\end{aligned}
$$

where $R_{12}=R_{1} \| R_{2}$. The inverse Laplace transform of Equation $\mathrm{S} 10$ will produce the analytical time-domain solution $i_{1}(t)$. To do this, the denominator must be expanded into its individual terms, such that:

$$
\begin{aligned}
\frac{\beta}{R_{1}}\left[\frac{s+\frac{1}{R_{2} C}}{s^{2}\left(s+\frac{1}{R_{12} C}\right)}\right] & =\frac{E+F s}{s^{2}}+\frac{G}{\left(s+\frac{1}{R_{12} C}\right)} \\
& =\frac{E}{s^{2}}+\frac{F}{s}+\frac{G}{\left(s+\frac{1}{R_{12} C}\right)}
\end{aligned}
$$

where $E, F$ and $G$ are hypothetical constants that will, by the rules of partial fractions, produce the original numerator, i.e. such that:

$$
(E+F s)\left(s+\frac{1}{R_{12} C}\right)+G s^{2}=\frac{\beta}{R_{1}}\left(s+\frac{1}{R_{2} C}\right)
$$

By reference to the table of selected Laplace functions presented in Table S1, it can be seen that the right hand side of Equation S12 corresponds to the summation of a ramp, a step and an exponential decay.

\begin{tabular}{ccc}
\hline $\begin{array}{c}\text { Function in } \\
\text { s-domain }\end{array}$ & Description & $\begin{array}{c}\text { Function in } \\
\text { time-domain }\end{array}$ \\
$1 / s^{2}$ & Ramp & $t u(t)$ \\
\hline $1 / s$ & Step & $u(t)$ \\
\hline $1 /(s+\alpha)$ & Exponential Decay & $\exp (-\alpha t) u(t)$ \\
\hline
\end{tabular}

Table S1: Table of selected Laplace functions and their time-domain equivalents

If $E, F$ and $G$ can successfully combine to produce the numerator, then the terms in units, $s$ and $s^{2}$ must agree, which produces three simultaneous solutions:

$$
\begin{array}{ll}
\text { terms in units } & \frac{E}{R_{12} C}=\frac{\beta}{R_{1} R_{2} C} \\
\text { terms in } s & E+\frac{F}{R_{12} C}=\frac{\beta}{R_{1}} \\
\text { terms in } s^{2} & F+G=0
\end{array}
$$


Rearranging Equation S14 produces:

$$
E=\frac{\beta R_{12} C}{R_{1} R_{2} C}=\frac{\beta}{R_{1}+R_{2}}
$$

which, on substituting into Equation S15 produces:

$$
\begin{aligned}
\frac{\beta}{R_{1}+R_{2}}+\frac{F}{R_{12} C} & =\frac{\beta}{R_{1}} \\
F & =\beta R_{12} C\left(\frac{1}{R_{1}}-\frac{1}{R_{1}+R_{2}}\right) \\
& =\beta C\left(\frac{R_{2}}{R_{1}+R_{2}}\right)^{2}
\end{aligned}
$$

Therefore the full analytical solution of the transient response of the RCR-network shown in Figure S1a to a ramp input of slope $\beta$ in $\mathrm{Vs}^{-1}$ is:

$$
\begin{aligned}
i_{1}(t) & =\left[E t+F+G \exp \left(\frac{-t}{T}\right)\right] u(t) \\
\text { where } E & =\frac{\beta}{R_{1}+R_{2}} \\
F & =\beta C\left(\frac{R_{2}}{R_{1}+R_{2}}\right)^{2} \\
G & =-F \\
T & =\left(R_{1} \| R_{2}\right) C
\end{aligned}
$$

and $u(t)$ is a unit step function at time $t=0$.

\section{S2. RCR Network: reverse derivation}

In order to calculate the values of $C, R_{1}$ and $R_{2}$ to which any given set of the parameters $E, F, T$ and $\beta$ correspond, Equations S22, S23 and S25 can be rewritten as, for example:

$$
\begin{aligned}
R_{1}+R_{2} & =\beta / E \\
\frac{R_{2}}{R_{1}+R_{2}} & =\sqrt{\frac{F}{\beta C}} \\
\left(R_{1} \| R_{2}\right) C=\frac{R_{1} R_{2} C}{R_{1}+R_{2}} & =T
\end{aligned}
$$

Substituting Equations S27 into S28, and S26 into S27 produces:

$$
\begin{aligned}
& R_{1} C \sqrt{\frac{F}{\beta C}}=T \quad \text { therefore } \quad R_{1}=\frac{T}{F} \sqrt{\frac{\beta F}{C}} \\
& R_{2} \frac{E}{\beta} \quad=\sqrt{\frac{F}{\beta C}} \quad \text { therefore } \quad R_{2}=\frac{1}{E} \sqrt{\frac{\beta F}{C}}
\end{aligned}
$$


which can be substituted into Equation S26 to produce:

$$
\begin{aligned}
\frac{\beta}{E} & =\frac{T}{F} \sqrt{\frac{\beta F}{C}}+\frac{1}{E} \sqrt{\frac{\beta F}{C}} \\
\sqrt{C} & =\frac{E}{\beta} \sqrt{\beta F}\left[\frac{T}{F}+\frac{1}{E}\right] \\
\text { therefore } \quad C & =\frac{(E T+F)^{2}}{\beta F}
\end{aligned}
$$

Once a value for $C$ has been calculated using Equation $\mathrm{S} 33$, values for $R_{1}$ and $R_{2}$ can be calculated directly using Equations S29 and S30.

\section{S3. RCRCR Network: Derivation of response to a voltage ramp.}

The addition of an extra RC-network to the Randles equivalent circuit produces the circuit shown in Figure S2a, and for the s-domain as shown in Figure S2b.

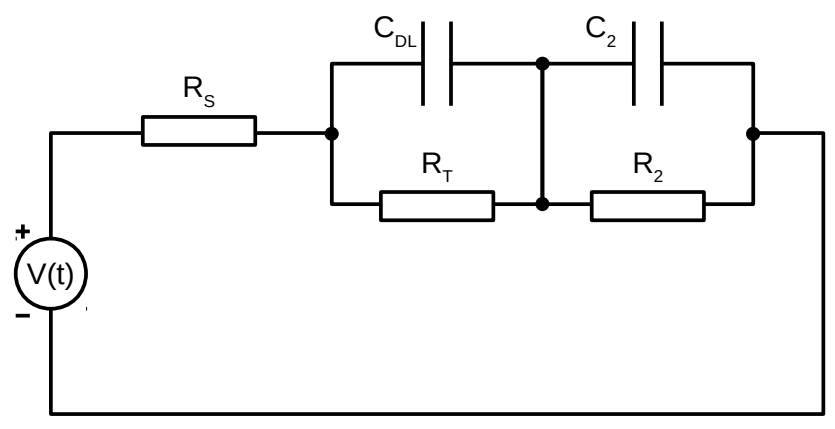

(a) Extended Randles electrical equivalent circuit

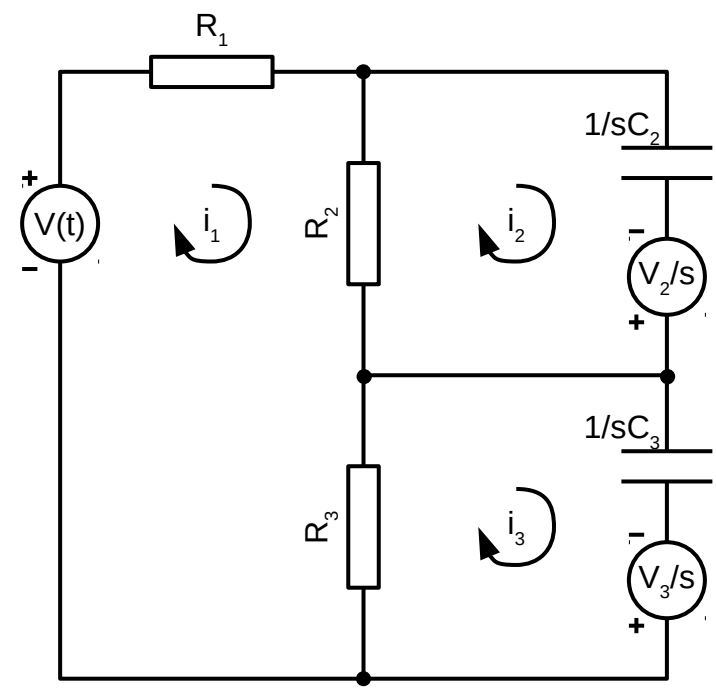

(b) As redrawn in s-domain

Figure S2: Equivalent circuit for the electrode/electrolyte interface extended to include an additional RC network

The result of performing mesh-current analysis on the rearranged circuit is the following three equations:

$$
\begin{aligned}
R_{1} i_{1}+R_{2}\left(i_{1}-i_{2}\right)+R_{3}\left(i_{1}-i_{3}\right) & =V(t) \\
\frac{i_{2}}{s C_{2}}+R_{2}\left(i_{2}-i_{1}\right) & =\frac{V_{2}}{s} \\
\frac{i_{3}}{s C_{3}}+R_{3}\left(i_{3}-i_{1}\right) & =\frac{V_{3}}{s}
\end{aligned}
$$

Assuming that the charge on the capacitors at time $t=0$ is $0 \mathrm{~V}$, which means that $V_{2}=V_{3}=0$, equations S35 and S36 can be rewritten as:

$$
\begin{aligned}
i_{2} & =\left(\frac{s}{s+\omega_{2}}\right) i_{1} \\
i_{3} & =\left(\frac{s}{s+\omega_{3}}\right) i_{1}
\end{aligned}
$$


where $\omega_{2}=\frac{1}{R_{2} C_{2}}$ and $\omega_{3}=\frac{1}{R_{3} C_{3}}$. Substituting equations S37 and S38 into equation S34 produces:

$$
\begin{aligned}
\left(R_{1}+R_{2}+R_{3}\right) i_{1}-R_{2}\left(\frac{s}{s+\omega_{2}}\right) i_{1}-R_{3}\left(\frac{s}{s+\omega_{3}}\right) i_{1} & =\frac{\beta}{s^{2}} \\
{\left[\frac{\left(R_{1}+R_{2}+R_{3}\right)\left(s+\omega_{2}\right)\left(s+\omega_{3}\right)-R_{2} s\left(s+\omega_{3}\right)-R_{3} s\left(s+\omega_{2}\right)}{\left(s+\omega_{2}\right)\left(s+\omega_{3}\right)}\right] i_{1} } & =\frac{\beta}{s^{2}} \\
{\left[R_{1} s^{2}+\left[\left(R_{1}+R_{2}\right) \omega_{2}+\left(R_{1}+R_{3}\right) \omega_{3}\right] s+\left(R_{1}+R_{2}+R_{3}\right) \omega_{2} \omega_{3}\right] } & i_{1}=\beta \frac{\left(s+\omega_{2}\right)\left(s+\omega_{3}\right)}{s^{2}}
\end{aligned}
$$

where $\beta / s^{2}$ is the Laplace transform of the driving voltage waveform, which is defined as a ramp starting at time $t=0$ with slope $\beta$ measured in $\mathrm{Vs}^{-1}$. If it is assumed that the left side can be factored, this can be rewritten as:

$$
i_{1}=\beta \frac{\left(s+\omega_{2}\right)\left(s+\omega_{3}\right)}{s^{2}\left(s+\alpha_{1}\right)\left(s+\alpha_{2}\right)}
$$

where $-\alpha_{1}$ and $-\alpha_{2}$ are the roots of the quadratic equation $a s^{2}+b s+c$, such that:

$$
\begin{aligned}
\alpha_{1} & =\frac{b-\sqrt{b^{2}-4 a c}}{2 a} \\
\alpha_{2} & =\frac{b+\sqrt{b^{2}-4 a c}}{2 a} \\
\text { where } \quad a & =R_{1} \\
b & =\left(R_{1}+R_{2}\right) \omega_{2}+\left(R_{1}+R_{3}\right) \omega_{3} \\
\text { and } \quad c & =\left(R_{1}+R_{2}+R_{3}\right) \omega_{2} \omega_{3}
\end{aligned}
$$

The condition for the existence of (real) solutions to the quadratic $\left(b^{2}>=4 a c\right)$ has been investigated numerically and found to be generally true, but efforts to demonstrate this mathematically have not proved successful. It is expected to be true, since any arbitrary network of resistors and capacitors cannot produce oscillatory behaviour. As before, the inverse Laplace transform of Equation S39 will produce the analytical time-domain solution $i_{1}(t)$. To do this, the denominator must be expanded into its individual terms, such that:

$$
\beta \frac{\left(s+\omega_{2}\right)\left(s+\omega_{3}\right)}{s^{2}\left(s+\alpha_{1}\right)\left(s+\alpha_{2}\right)}=\frac{E+F s}{s^{2}}+\frac{G}{s+\alpha_{1}}+\frac{H}{s+\alpha_{2}}
$$

where $E, F, G$ and $H$ are constants. With reference to Table $\mathrm{S} 1$ it is informative to note that the solution is equal to the sum of a ramp, a step, and two exponential decays, and will therefore be of the form:

$$
i_{1}(t)=E t+F+G \exp \left(-\alpha_{1} t\right)+H \exp \left(-\alpha_{2} t\right)
$$

The constants $E$ through $H$ must be chosen such that, by the rules of partial fractions, they produce the original numerator, specifically:

$$
\begin{aligned}
& (E+F s)\left(s+\alpha_{1}\right)\left(s+\alpha_{2}\right)+G s^{2}\left(s+\alpha_{2}\right)+H s^{2}\left(s+\alpha_{1}\right)=\beta\left(s+\omega_{2}\right)\left(s+\omega_{3}\right) \\
& (F+G+H) s^{3}+\left(E+F\left(\alpha_{1}+\alpha_{2}\right)+G \alpha_{2}+H \alpha_{1}\right) s^{2}+ \\
& \left(E\left(\alpha_{1}+\alpha_{2}\right)+F \alpha_{1} \alpha_{2}\right) s+E \alpha_{1} \alpha_{2}=\beta\left[s^{2}+\left(\omega_{2}+\omega_{3}\right) s+\omega_{2} \omega_{3}\right]
\end{aligned}
$$


which produces four simultaneous equations for the matching terms in $s^{3}, s^{2}, s$ and units:

$$
\begin{aligned}
F+G+H & =0 \\
E+F\left(\alpha_{1}+\alpha_{2}\right)+G \alpha_{2}+H \alpha_{1} & =\beta \\
E\left(\alpha_{1}+\alpha_{2}\right)+F \alpha_{1} \alpha_{2} & =\beta\left(\omega_{2}+\omega_{3}\right) \\
E \alpha_{1} \alpha_{2} & =\beta \omega_{2} \omega_{3}
\end{aligned}
$$

multiplying equation S46 by $\alpha_{1}$ and subtracting from equation S47 produces:

$$
E+F \alpha_{2}+G\left(\alpha_{2}-\alpha_{1}\right)=\beta
$$

therefore $E, F, G$ and $H$ can be calculated in the sequence:

$$
\begin{aligned}
E & =\frac{\beta \omega_{2} \omega_{3}}{\alpha_{1} \alpha_{2}} \\
F & =\frac{\beta\left(\omega_{2}+\omega_{3}\right)-E\left(\alpha_{1}+\alpha_{2}\right)}{\alpha_{1} \alpha_{2}} \\
G & =\frac{\beta-E-F \alpha_{2}}{\alpha_{2}-\alpha_{1}} \\
H & =-(F+G)
\end{aligned}
$$

Example. Let the component values of the equivalent circuit be assigned as follows: $R_{1}=1 \Omega, R_{2}=237 \Omega, C_{2}=1830 \mu \mathrm{F}$, $R_{3}=4180 \Omega, C_{3}=1830 \mu \mathrm{F}$. The calculation of the response will therefore proceed as follows:

$$
\begin{aligned}
\omega_{2} & =2.306 \mathrm{~Hz} \\
a & =1 \\
c & =1332 \\
\alpha_{1} & =1.217 \mathrm{~Hz} \\
E & =2.263 \mu \mathrm{As}^{-1} \\
G & =-7.304 \mu \mathrm{A}
\end{aligned}
$$

$$
\begin{aligned}
\omega_{3} & =0.1307 \mathrm{~Hz} \\
b & =1095 \\
b^{2}-4 a c & =1194427 \\
\alpha_{2} & =1094 \mathrm{~Hz} \\
F & =16.43 \mu \mathrm{A} \\
H & =-9.130 \mu \mathrm{A}
\end{aligned}
$$

With reference to Equation S45, the analytical response of the 5-component circuit to a voltage ramp of $10 \mathrm{mV} \mathrm{s}^{-1}$ can therefore be plotted, as presented in Figure S3a. The figure includes the results of a Spice simulation, which serve to verify that the two methods are in agreement. The detail view of the first $10 \mathrm{~ms}$ in Figure S3b confirms the presence of an initial rapid exponential decay, which is followed by the a slower decay that takes several seconds. The plot is therefore exhibiting the bi-exponential behaviour predicted from the analytical solution.

\section{S4. Simplification if solution resistance is small}

If $R_{1}$ is small compared to both $R_{2}$ and $R_{3}$, then the definitions of $b$ and $c$ can be simplified to:

$$
\begin{aligned}
& b \approx R_{2} \omega_{2}+R_{3} \omega_{3}=1 / C_{2}+1 / C_{3}=1 / C_{23} \\
& c \approx\left(R_{2}+R_{3}\right) \omega_{2} \omega_{3}=\frac{R_{2}+R_{3}}{R_{2} R_{3} C_{2} C_{3}}=\frac{1}{R_{23} C_{2} C_{3}}
\end{aligned}
$$




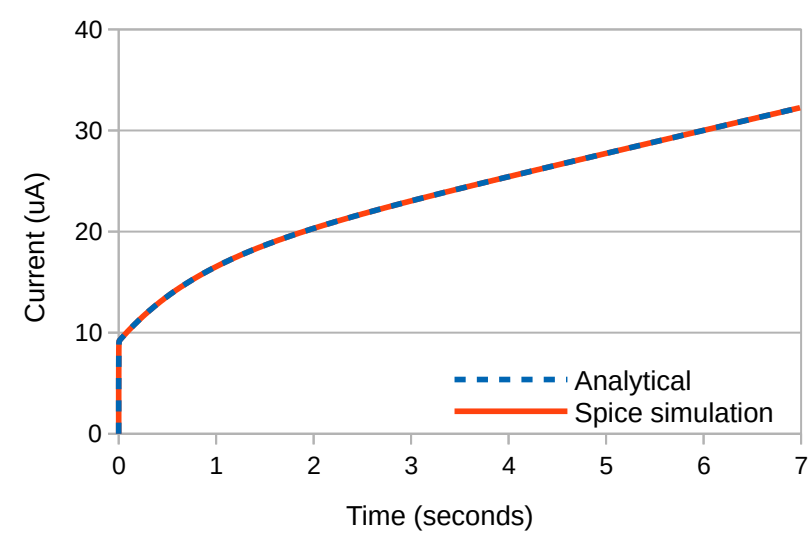

(a) Full view

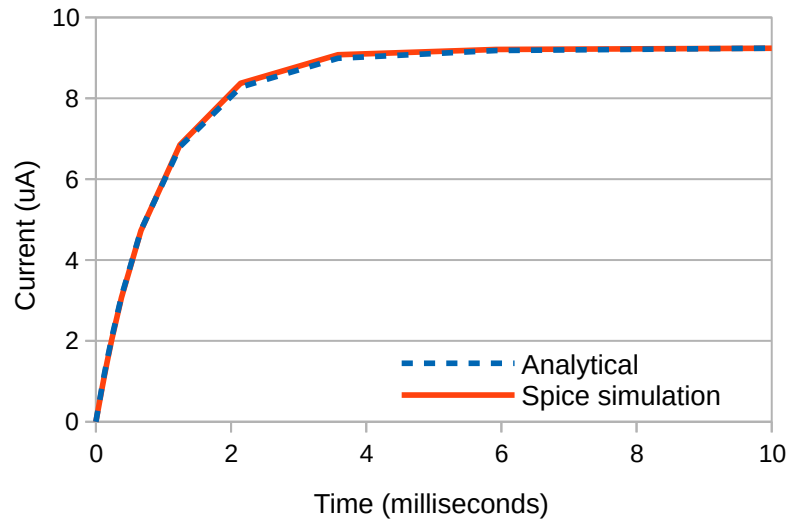

(b) Detail view of the first $10 \mathrm{~ms}$

Figure S3: Transient response of the 5-component extended Randles equivalent circuit to a $10 \mathrm{mV} \mathrm{s}^{-1}$ voltage ramp.

where $C_{23}=C_{2} \| C_{3}$ and $R_{23}=R_{2} \| R_{3}$. This means that $b^{2}-4 a c$ becomes:

$$
b^{2}-4 a c=\left(\frac{1}{C_{23}}\right)^{2}-\frac{4 R_{1}}{R_{23} C_{2} C_{3}}
$$

which, if $R_{1}$ is small, becomes just $b^{2}$, which in turn means that Equation S40 becomes:

$$
\alpha_{2} \approx \frac{b+\sqrt{b^{2}}}{2 a}=\frac{b}{a}=\frac{1}{R_{1} C_{23}}
$$

This means that the time-constant of the fast exponential decay is $\approx R_{1} C_{23}$, or the combination of the solution resistance $R_{S}$ and the two capacitances in series. This is understandable, since initially both capacitors are discharged (or at equilibrium) and their resistance is low. However, they quickly adopt voltages which resist further current conduction, and a slower charging process ensues. To calculate the second time-constant, it is helpful to rewrite the square root as a binomial expansion:

$$
(1+x)^{n}=1+\frac{n}{1 !} x+\frac{n(n-1)}{2 !} x^{2}+\ldots
$$

therefore Equation S41 becomes:

$$
\begin{aligned}
\alpha_{1} & =\frac{b-b\left[1-\frac{4 a c}{b^{2}}\right]^{1 / 2}}{2 a} \\
& =\frac{b-b\left[1-\frac{1}{2} \frac{4 a c}{b^{2}}-\frac{1}{4}\left(\frac{4 a c}{b^{2}}\right)^{2}-\ldots\right]}{2 a}
\end{aligned}
$$

Ignoring all but the first and second terms of the expansion, this simplifies to:

$$
\alpha_{1} \approx \frac{c}{b}=\frac{1}{R_{23}\left(C_{2}+C_{3}\right)}
$$

This means that the time-constant of the slow exponential decay is $\approx R_{23}\left(C_{2}+C_{3}\right)$. This makes sense, since it is a time-constant derived from the two RC-networks combined. The ratio of the two time-constants is therefore equal to:

$$
\frac{\alpha_{2}}{\alpha_{1}}=\frac{R_{23}\left(C_{2}+C_{3}\right)}{R_{1} C_{23}}
$$


For any given values of $C_{3}, R_{1}, R_{2}$ and $R_{3}$, this reaches a minimum where $C_{2}=C_{3}$ of:

$$
\frac{\alpha_{2}}{\alpha_{1}}=\frac{4 R_{23}}{R_{1}}
$$

therefore, regardless of the relative values of capacitance, the ratio of the two time-constants will always be large if $R_{1}$ is small compared with both $R_{2}$ and $R_{3}$.

\section{S5. RCRCR Network: Measurable quantities}

Given that one of the time-constants for the two exponential decays is so rapid, it is unlikely that it can be accurately measured using CV. Similarly, given that the other time-constant is so slow, it is unlikely that it can be accurately measured using EIS. However, if it is possible to accurately measure the fast time-constant using EIS, and the slow one using $\mathrm{CV}$, information gained from both the time and frequency domains could be combined to obtain a solution.

Accurate measurements can also be obtained for $E$ (the current ramp rate), $R_{1}$ (the solution resistance) and the sum of $G$ and $H$ (the magnitude of the two exponential decays added together). From Equation S46 it is seen that:

$$
G+H=-F
$$

therefore the sum of $G$ and $H$ actually provides the value of $F$. Hence the measurable parameters are:

$$
\alpha_{1}, \alpha_{2}, E, R_{1} \text { and } F
$$

Given that there are five components in the RCRCR network, this means that it should be possible to determine the values of all five. This is assuming that the five measurable parameters are sufficiently independent, and that the solution space is suitably shaped and unambiguous.

From inspection of Equations S40 and S41, it can be seen that:

$$
\alpha_{1} \alpha_{2}=\frac{c}{a} \quad \text { and } \quad \alpha_{1}+\alpha_{2}=\frac{b}{a}
$$

Therefore the definition of $E$ from Equation S48 may be rewritten as:

$$
\begin{aligned}
E & =\beta \omega_{2} \omega_{3} \frac{a}{c} \\
E & =\beta \omega_{2} \omega_{3}\left[\frac{R_{1}}{\left(R_{1}+R_{2}+R_{3}\right) \omega_{2} \omega_{3}}\right] \\
\text { therefore } \quad R_{2}+R_{3} & =k_{1} \\
\text { where } \quad k_{1} & =\frac{\beta}{E} R_{1}-R_{1}=R_{1}(\beta / E-1)
\end{aligned}
$$

where the definitions of $a$ and $c$ from Equations S42 and S44 have been used. Since all of the quantities on the right hand side are measurable, this means that if $R_{2}$ is known, then $R_{3}$ can be calculated, which immediately reduces the size of the solution space by one dimension. Assuming $R_{1}$ is small, and given that $C_{23}=C_{2} \| C_{3}$, the simplified definition of $\alpha_{2}$ from Equation S51 can be rewritten as:

$$
\begin{aligned}
C_{2} C_{3} R_{1} \alpha_{2} & =C_{2}+C_{3} \\
\text { therefore } \quad C_{3} & =\frac{C_{2}}{k_{2} C_{2}-1} \\
\text { where } \quad k_{2} & =\alpha_{2} R_{1}
\end{aligned}
$$


which means that if $C_{2}$ is known, then $C_{3}$ can be calculated. Given that $R_{1}$ is measurable, this means that the potentially five-dimensional solution space has been reduced to just two dimensions. The definition of $F$ from Equation S49 describes another of the measurable quantities, and can be rewritten as:

$$
\begin{aligned}
\frac{1}{R_{2} C_{2}}+\frac{1}{R_{3} C_{3}} & =\frac{F \alpha_{1} \alpha_{2}+E\left(\alpha_{1}+\alpha_{2}\right)}{\beta} \\
\text { therefore } \quad R_{2} C_{2}+R_{3} C_{3} & =k_{3} R_{2} C_{2} R_{3} C_{3} \\
\text { where } \quad k_{3} & =\left[F \alpha_{1} \alpha_{2}+E\left(\alpha_{1}+\alpha_{2}\right)\right] / \beta
\end{aligned}
$$

The final measurable quantity, $\alpha_{1}$, can be incorporated by rewriting Equation S52 as:

$$
\begin{aligned}
R_{23}\left(C_{2}+C_{3}\right) & =1 / \alpha_{1} \\
\text { therefore } \quad R_{2} R_{3}\left(C_{2}+C_{3}\right) & =k_{4} \\
\text { where } \quad k_{4} & =k_{1} / \alpha_{1}
\end{aligned}
$$

This produces four simultaneous equations with four unknowns:

$$
\begin{aligned}
R_{2}+R_{3} & =k_{1} \\
C_{3} & =C_{2} /\left(k_{2} C_{2}-1\right) \\
R_{2} C_{2}+R_{3} C_{3} & =k_{3} R_{2} C_{2} R_{3} C_{3} \\
R_{2} R_{3}\left(C_{2}+C_{3}\right) & =k_{4} \\
\text { where } \quad k_{1} & =R_{1}(\beta / E-1) \\
k_{2} & =\alpha_{2} R_{1} \\
k_{3} & =\left[F \alpha_{1} \alpha_{2}+E\left(\alpha_{1}+\alpha_{2}\right)\right] / \beta \\
k_{4} & =k_{1} / \alpha_{1}
\end{aligned}
$$

and where two of the pairs of unknowns are directly related. All parameters $k_{n}$ are defined solely in terms of measurable parameters. It therefore looks feasible to solve the above equations and (in combination with $R_{1}$ ) to determine all of the component values in the electrical equivalent circuit. However, attempts to solve the above equations analytically produced a quartic equation, therefore numerical and graphical methods were employed.

Example. Experiments on a smooth TiN-coated electrode produced the CV and EIS results presented in Figure 6 of the main manuscript. Note that the CV response does indeed show evidence of bi-exponential behaviour, and with widely varying time-constants. Although this is not always so clear, it is useful confirmation that the electrochemical system exhibits behaviour which directly supports the proposed 5-element equivalent circuit. In any case, it is not possible for a simple 3-element model to match the observed EIS and CV behaviour of any of the electrodes.

From the EIS results the value of $R_{1}$ is determined by measuring the point of closest approach to the origin at high frequency, and the value of $\alpha_{2}$ is determined by measuring the position of the main breakpoint. This is defined as the frequency (in radians per second) at which the impedance magnitude reaches $\sqrt{2} R_{1}$, and is highlighted by the vertical dashed line in Figure 7b. The values of $E, F$ and $T$ were determined using best-fit RCR-network transient curve fitting, as performed at https://fitting.gannon.me.uk. For curves exhibiting a bi-exponential decay, more accurate values of $E, F$ and $T$ were measured by offsetting the measurement window by two sample points $(100 \mathrm{~ms})$, with the values measured as presented in Table 3 of the main manuscript. An estimate of the value of $H$ (taken from the second sample point) was added to the fitted value of $F$ to generate an overall target value of $F$, as shown in the bottom row of the table.

Using the measured values, and employing the simplification that $R_{1} \ll R_{2}$ and $R_{3}$, it is possible to explore the 
resulting two-dimensional solution space using a suitable cost function, as presented in Figure S4. In this case the cost-function chosen was:

$$
\begin{aligned}
e r r_{1} & =R_{2} C_{2}+R_{3} C_{3}-k_{3} R_{2} C_{2} R_{3} C_{3} \\
e r r_{2} & =R_{2} R_{3}\left(C_{2}+C_{3}\right)-k_{4} \\
\text { cost } & =\log \left(e r r_{1}^{2} \times e r r_{2}^{2}\right)
\end{aligned}
$$

which is derived directly from Equations S57 and S58. This technique identifies the values of $R_{2}$ and $C_{2}$ which constitute the best-fit to the EIS and CV data, as highlighted by the black circle. Since $R_{1}$ is already known, and (in the simplified solution space) $R_{3}$ can be calculated from $R_{2}$, and $C_{3}$ from $C_{2}$, this therefore identifies the full best-fit 5-element RCRCR network, as presented in Table $\mathrm{S} 2$ in the column headed '2D Values'.

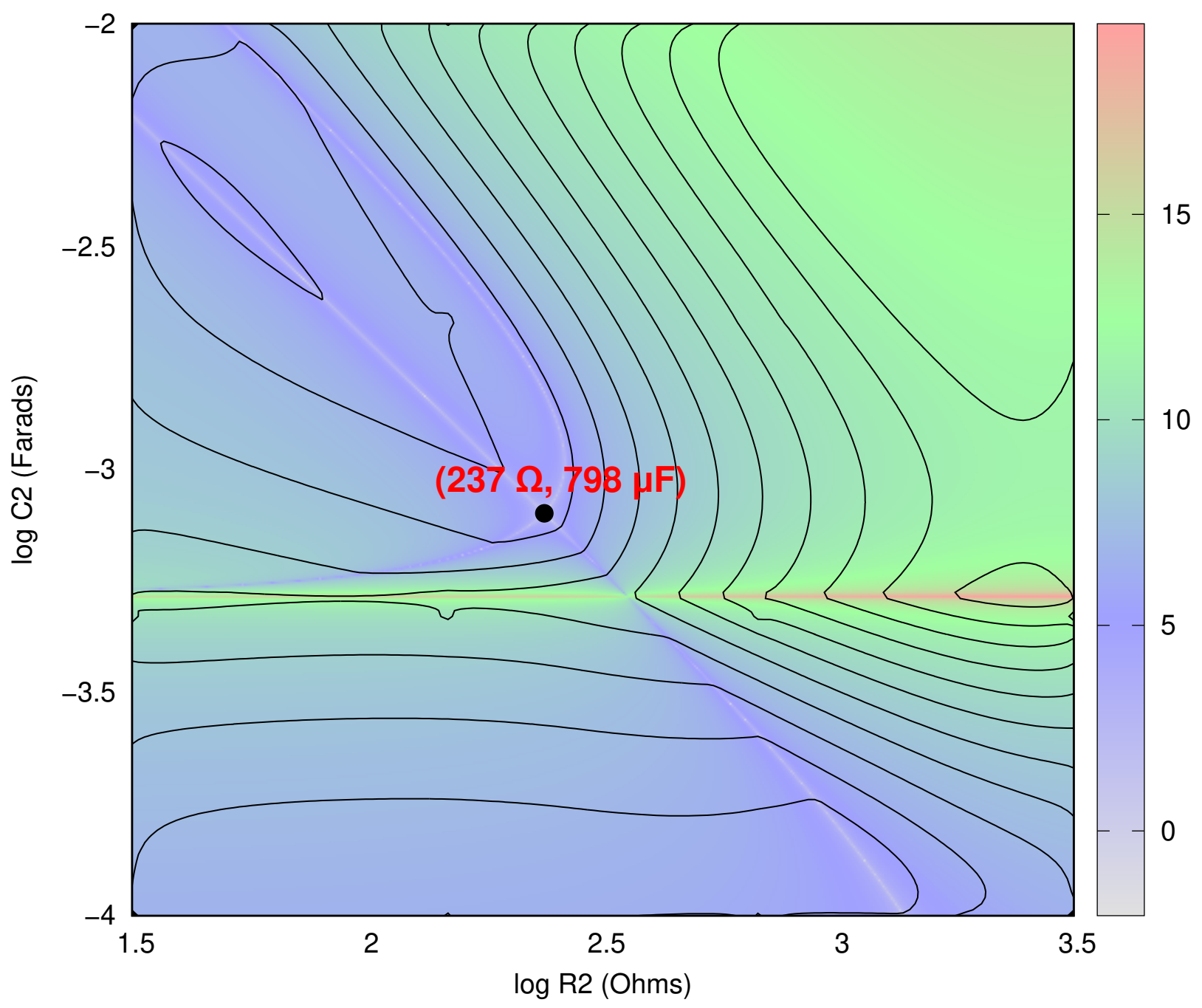

Figure S4: Plot of cost function for the simplified two-dimensional solution space, based on the assumption that $R_{1} \ll R_{2}$ and $R_{3}$. The above point of best match is then used as the starting point for conventional four-dimensional gradient descent.

Cross-checking the values of $E$ and $F$ produced by the 2D best-fit 5-element network using Equations S48 and S49 reveals that this procedure produces a value of $F$ that is too small. Its transient response is therefore a poor match to the 
measured CV waveform, and this can be attributed to the over-simplification of the solution space to two-dimensions. Nevertheless, the 2D best-fit can be used as a starting point from which to employ conventional gradient descent within the full four-dimensional solution space. It should be noted that because the curvature the solution space is not conducive to gradient descent, it is observed that the use of a random starting point does not succeed in finding a solution.

\begin{tabular}{|c|l|l|}
\hline Parameter & 2D Values & 4D Values \\
\hline $\mathbf{R}_{\mathbf{1}}$ & $0.778 \Omega$ & $0.778 \Omega$ \\
\hline $\mathbf{R}_{\mathbf{2}}$ & $237 \Omega$ & $118 \Omega$ \\
\hline $\mathbf{C}_{\mathbf{2}}$ & $800 \mu \mathrm{F}$ & $2100 \mu \mathrm{F}$ \\
\hline $\mathbf{R}_{\mathbf{3}}$ & $5490 \Omega$ & $5610 \Omega$ \\
\hline $\mathbf{C}_{\mathbf{3}}$ & $1500 \mu \mathrm{F}$ & $2420 \mu \mathrm{F}$ \\
\hline
\end{tabular}

Table S2: Best-fit values obtained for the 5-element equivalent circuit model matching the measurement data presented in Figure 6. Column 2 presents the values obtained within the simplified two-dimensional solution space, and column 3 those obtained after using column 2 as a starting point for full four-dimensional gradient descent.

The values produced for the 5-element equivalent electrical circuit as a result of four-dimensional gradient descent are as presented in Table S2 in column '4D Values'. The descent algorithm used was Barzilai-Borwein, and the cost function was defined as:

$$
\operatorname{cost}=\log \left(\left[\log \left(E / E_{T}\right)\right]^{2}+\left[\log \left(F / F_{T}\right)\right]^{2}+\left[\log \left(\alpha_{1} / \alpha_{1 T}\right)\right]^{2}+\left[\log \left(\alpha_{2} / \alpha_{2 T}\right)\right]^{2}\right)
$$

where $E_{T}, F_{T}, \alpha_{1 T}$ (which equals $1 / T_{T}$ ) and $\alpha_{2 T}$ are the target measured values from Table 3 . These gradient-descent values constitute the best-known fit to the data.

\section{S6. Constant Phase Element: Transient Simulation}

The general equation for the impedance of a constant phase element (CPE) is:

$$
Z(\omega)=\frac{1}{Q}\left[\frac{1}{(j \omega)^{\alpha}}\right]
$$

where $Q$ is the magnitude of the CPE, and $\alpha$ is its argument, such that $0 \leq \alpha \leq 1$. A Warburg element is simply a CPE where the argument is equal to 0.5 .

$$
Z(\omega)=\frac{1}{Q}\left[\frac{1}{\sqrt{j \omega}}\right]
$$

As a function of time, the voltage across the CPE is given by a convolution integral:

$$
V_{C P E}(t)=\frac{1}{Q \Gamma(\alpha)} \int_{0}^{t}(t-u)^{\alpha-1} I(u) d u
$$

where $\Gamma(\alpha)$ is the gamma function [41]. It can thus be seen for an ideal capacitor (where $\alpha=1)$ that $(t-u)^{\alpha-1}=\Gamma(\alpha)=1$, and the equation simplifies to:

$$
V_{C}(t)=\frac{1}{C} \int I d t
$$

which can be recognised as the standard formula for a capacitor. However, Equation S61 provides the voltage as a function of the current, which is inconvenient in voltammetry where it is the voltage that is controlled and the current that is to be measured. Solving this involves expressing the electrical network as a differential equation.

For example, for the basic RC network shown in Figure S5 the equation can be expressed as:

$$
V_{\text {Total }}=\frac{d q}{d t} R+\frac{q}{C}
$$




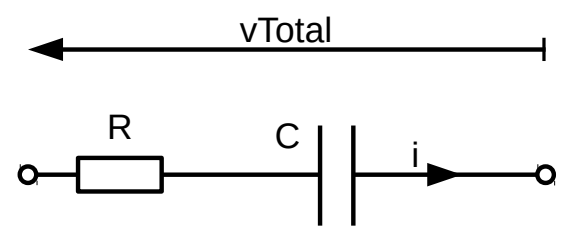

Figure S5: RC Network

where $q$ is electrical charge, such that $i=d q / d t$. Since $q / C$ is equal to the voltage on the capacitor, $V_{C}$, this can be expressed more generally as:

$$
V_{\text {Total }}=\frac{d q}{d t} R+V_{C}
$$

The Euler method can be used to approximate a solution to this equation, based on the first-order simplification that:

$$
q_{n+1}=q_{n}+h \frac{d q}{d t}
$$

where $h$ is the step size in time. Replacing the capacitor by a CPE, and therefore $V_{C}$ by $V_{C P E}$, produces:

$$
q_{n+1}=q_{n}+h\left(\frac{V_{\text {Total }}-V_{C P E}}{R}\right)
$$

This iterative scheme can be converted into a computer program, as shown in Listing 1, where the language chosen was PHP. When executed, this produced the simulation results presented in Figure S6a.

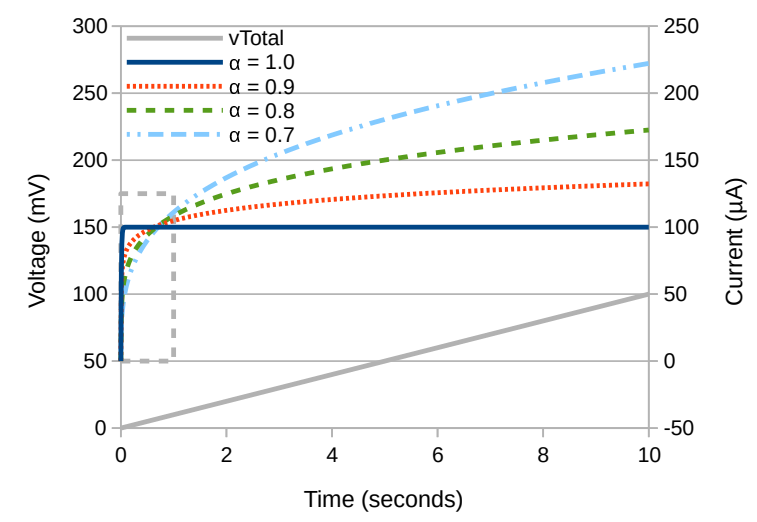

(a) Transient simulation

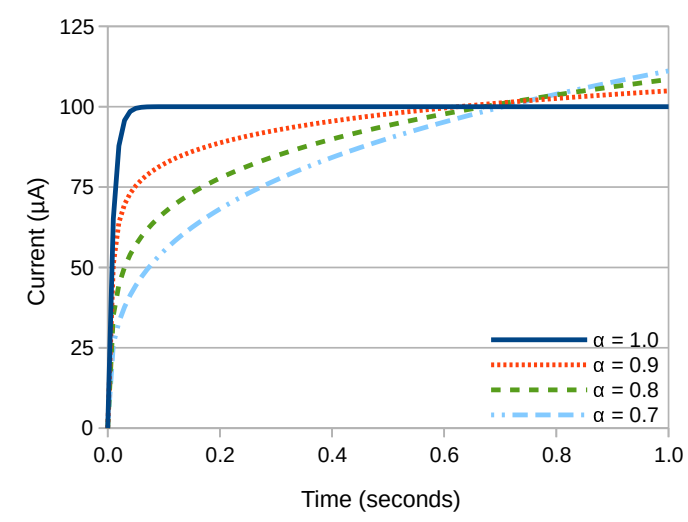

(b) Detail view of dashed gray box

Figure S6: Transient simulation of an RQ network for 4 different values of $\alpha . R=1 \Omega, Q=0.01$.

The results show that as the argument of the CPE decreases, its leakage increases, and so too does the total amount of current. The asymptotic response of the circuit towards a fixed current also changes, and is instead replaced by a more curved response. The RQ network is thus able to emulate the behaviour of a much higher resistance, even though no such resistance is present.

The detailed view of the dashed gray box in Figure S6b shows that when $\alpha=1$, the current reaches the asymptotic value of $100 \mu \mathrm{A}$ rapidly in less than $100 \mathrm{~ms}$. For $\alpha=0.9$, the response appears to be bi-exponential, so the RQ network is therefore exhibiting two separate time-constants. Irrespective of value, all traces converge after about $0.6 \mathrm{~s}$. However, the current does not asymptote towards a slope, which is the typical behaviour of an RCR network. 
For an RQR network ( $R_{1}$ in series, $R_{2}$ in parallel) the iterative scheme can be altered to become:

$$
q_{n+1}=q_{n}+\frac{h}{R_{1}}\left(V_{\text {Total }}-\frac{R_{1}+R_{2}}{R_{2}} V_{C P E}\right)
$$

where $q$ is the charge on the CPE. The total current is then:

$$
i(t)=\frac{d q}{d t}+\frac{V_{C P E}}{R_{2}}
$$

Since the function presented in Equation S61 is a convolution integral, it is possible to plot the convolution function, as presented in Figure S7. This shows that the function is flat if $\alpha=1$, which means that none of the charge placed onto the capacitor is lost. For other values of $\alpha$ losses occur, but all of the functions converge at a time value of about $0.5 \mathrm{~s}$. It would thus appear that the CPE has a characteristic time-constant of its own, and one that is unrelated to its magnitude or any other circuit components.

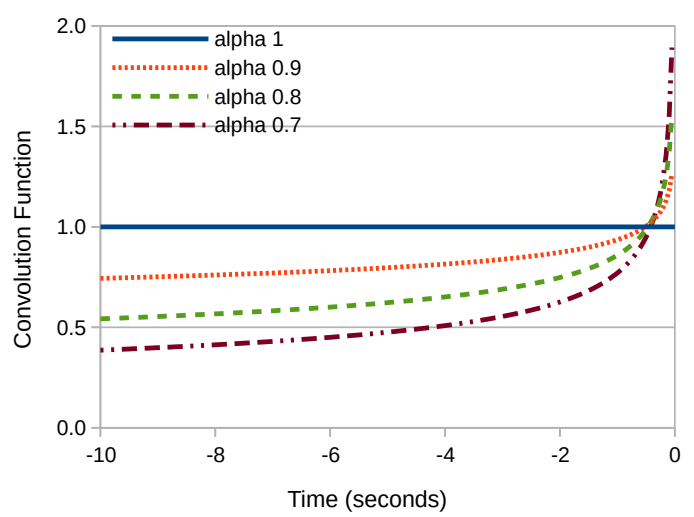

Figure S7: Convolution function used in the simulation of a constant phase element. 
Listing 1: PHP code to simulate the transient response of an RQ network to a voltage ramp.

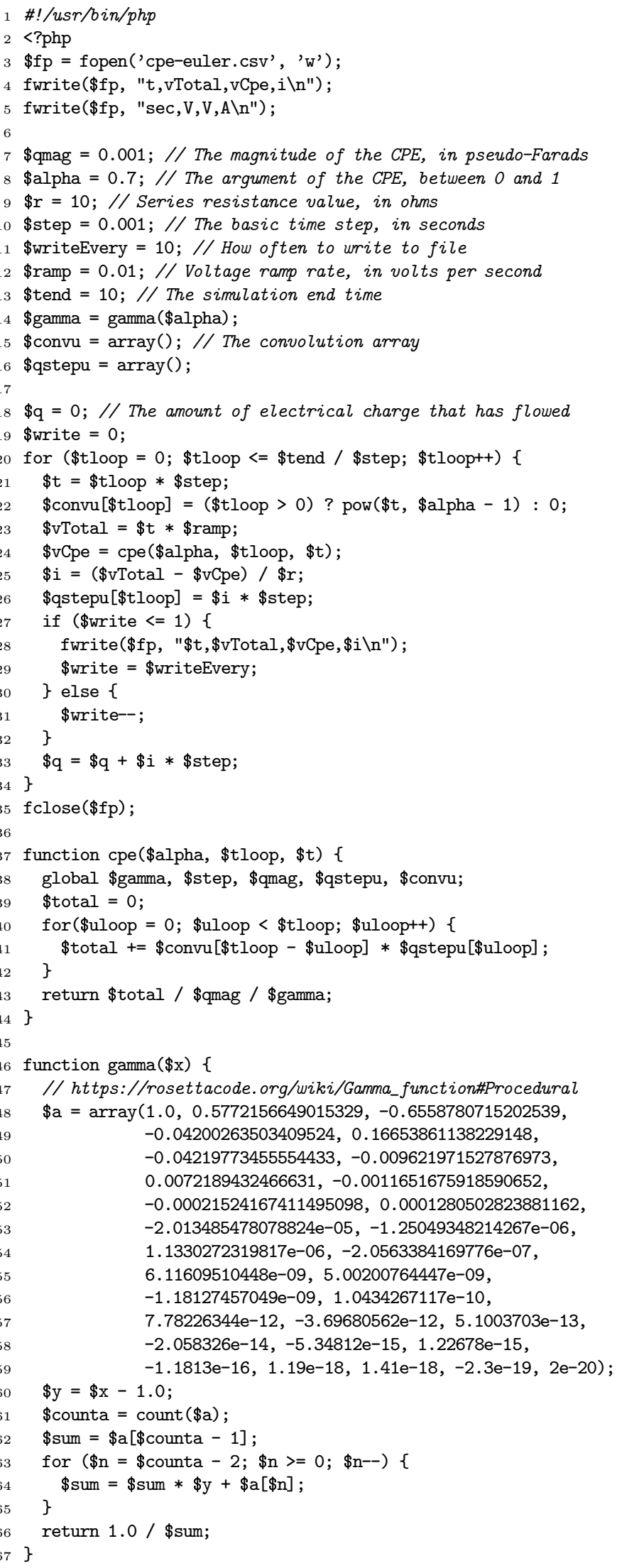

